Interfaces and Free Boundaries 16 (2014), 137-173

DOI 10.4171/IFB/316

\title{
Parabolic optimal control problems on evolving surfaces subject to point-wise box constraints on the control - theory and numerical realization
}

\author{
MORTEN VIERLING \\ Schwerpunkt Optimierung und Approximation, Universität Hamburg, Bundesstraße 55, 20146 \\ Hamburg, Germany \\ E-mail: morten.vierling@uni-hamburg.de
}

[Received 16 September 2011 and in revised form 30 September 2013]

\begin{abstract}
We consider control-constrained linear-quadratic optimal control problems on evolving hypersurfaces in $\mathbb{R}^{n+1}$. In order to formulate well-posed problems, we prove existence and uniqueness of weak solutions for the state equation, in the sense of vector-valued distributions. We then carry out and prove convergence of the variational discretization of a distributed optimal control problem. In the process, we investigate the convergence of a fully discrete approximation of the state equation, and obtain optimal orders of convergence under weak regularity assumptions. We conclude with a numerical example.
\end{abstract}

2010 Mathematics Subject Classification: Primary 58J35, 49J20, 49Q99, 35D30, 35R01.

Keywords: Evolving surfaces, weak solutions, parabolic optimal control, error estimates.

\section{Introduction}

We investigate parabolic optimal control problems on evolving material hypersurfaces in $\mathbb{R}^{n+1}$. Following [3], we consider a parabolic state equation in its weak form

$$
\frac{\mathrm{d}}{\mathrm{d} t} \int_{\Gamma(t)} y \varphi \mathrm{d} \Gamma(t)+\int_{\Gamma(t)} \nabla_{\Gamma} y \cdot \nabla_{\Gamma} \varphi \mathrm{d} \Gamma(t)=\int_{\Gamma(t)} y \dot{\varphi} \mathrm{d} \Gamma(t)+\int_{\Gamma(t)} f \varphi \mathrm{d} \Gamma(t),
$$

where $\Gamma=\{\Gamma(t)\}^{t \in[0, T]}$ is a family of $C^{2}$-smooth, compact $n$-dimensional surfaces in $\mathbb{R}^{n+1}$, evolving smoothly in time with velocity $V$. Further assume $f$ sufficiently smooth and let $\dot{\varphi}=$ $\partial_{t} \varphi+V \nabla \varphi$ denote the material derivative of a smooth test function $\varphi$.

We start by defining unique weak solutions for the state equation. The idea is to pull back the problem onto a fixed domain, introducing distributional material derivatives in the sense of [17] and a $W(0, T)$-like solution space. As a consequence, a large part of the theory developed around $W(0, T)$ for fixed domains applies, compare for example [17] and [16] .

An alternative approach to prove existence of weak solutions along the lines of [18] is taken in [22], that entirely avoids the notion of vector-valued distributions.

Recent works also deal with the discretization of (1.1), both in space, compare [4], and time, see [6] and [5].

In [4] order-optimal error bounds of type $\sup _{t \in[0, T]}\|\cdot\|_{L^{2}(\Gamma(t))}$ are derived for the discretization of the state equation, assuming a slightly higher regularity of the state than is used in Section 5 and 6 , where we derive $\left(\int_{0}^{T}\|\cdot\|_{L^{2}(\Gamma(t))}^{2} \mathrm{~d} t\right)^{\frac{1}{2}}$-like bounds. A class of Runge-Kutta methods to tackle the 
space-discretized problem is investigated in [6], assuming among other things that one can evaluate $f$ in a point-wise fashion, i.e. that $f(t) \in L^{2}(\Gamma(t))$ is well defined. For a fully discrete approach and the according error bounds see [5]. There a backwards Euler method is considered for time discretization whose implementation resembles our discontinuous Galerkin approach in Section 6. Yet while the approach in [5] ultimately leads to $\sup _{t \in[0, T]}\|\cdot\|_{L^{2}(\Gamma(t))}$-convergence, we allow for non-smooth controls and thus cannot expect to obtain such strong convergence estimates.

Basic facts on control constrained parabolic optimal control problems and their discretization can be found for example in [24] and [21], respectively.

The paper is structured as follows. We begin with a very short introduction into the setting in Section 2. In order to formulate well posed optimal control problems we first proof the existence of an appropriate weak solution in Section 3, complementing the existence results from [3]. We then use the the results from Section 3 in order to formulate control constrained optimal control problems in section 4. Afterwards, we examine the space- and time-discretization of the state equation in Sections 5 and 6, before returning to the optimal control problems in Section 7. There we apply variational discretization in the sense of [12] to achieve fully implementable optimization algorithms. We end the paper by giving a numerical example in Section 8 .

\section{Setting}

Before we can properly formulate (1.1), let us introduce some basic tools and clarify what our assumptions are regarding the family $\{\Gamma(t)\}_{t \in[0, T]}$.

Assumption 2.1 The hypersurface $\Gamma_{0}=\Gamma(0) \subset \mathbb{R}^{n+1}$ is $C^{2}$-smooth and compact (i.e. without boundary). $\Gamma$ evolves along a $C^{2}$-smooth velocity field $V: \mathbb{R}^{n+1} \times[0, T] \rightarrow \mathbb{R}^{n+1}$ with flow $\bar{\Phi}: \mathbb{R}^{n+1} \times[0, T]^{2} \rightarrow \mathbb{R}^{n+1}$, such that its restriction $\Phi_{t}^{s}(\cdot): \Gamma(s) \rightarrow \Gamma(t)$ is a diffeomorphism for every $s, t \in[0, T]$.

The assumption gives rise to a second representation of $\Gamma(t)$ and in particular implies $\Gamma(t)$ to be orientable with a smooth unit normal field $v(\cdot, t)$. As a consequence, the evolution of $\Gamma$ can be described as the level set of the signed distance function $d$ such that

$$
\Gamma(t)=\left\{x \in \mathbb{R}^{n+1} \mid d(x, t)=0\right\},
$$

as well as $|d(x, t)|=\operatorname{dist}(x, \Gamma(t))$ and $\nabla d(x, t)=v(x, t)$ for $x \in \Gamma(t)$. Further, we have $d(\cdot, t) \in$ $C^{2}\left(\eta_{r}(t)\right)$ for some tubular neighborhood $n_{r}(t)=\left\{x \in \mathbb{R}^{n+1}|| d(x, t) \mid \leqslant r\right\}$ of $\Gamma(t)$. Due to the uniform boundedness of the curvature of $\Gamma(t)$ the radius $r>0$ does not depend on $t \in[0, T]$. The domain of $d$ is $\eta_{T}=\bigcup_{t \in[0, T]} \eta_{r}(t) \times\{t\}$ which is a neighborhood of $\bigcup_{t \in[0, T]} \Gamma(t) \times\{t\}$ in $\mathbb{R}^{n+2}$.

Using $d$ we can define the projection

$$
a_{t}: \eta_{r}(t) \rightarrow \Gamma(t), \quad a_{t}(x)=x-d(x, t) \nabla d(x, t),
$$

which allows us to extend any function $\phi: \Gamma(t) \rightarrow \mathbb{R}$ to $\eta_{r}(t)$ by $\bar{\phi}(x)=\phi\left(a_{t}(x)\right)$. Hence we can represent the surface gradient in global exterior coordinates $\nabla_{\Gamma(t)} \phi=\left(I-v(\cdot, t) v(\cdot, t)^{T}\right) \nabla \bar{\phi}$ as the euclidean projection of the gradient of $\bar{\phi}$ onto the tangential space of $\Gamma(t)$. In the following we will write $\nabla_{\Gamma}$ instead of $\nabla_{\Gamma(t)}$, wherever it is clear which surface $\Gamma(t)$ the gradient relates to.

We are going to exploit existing results on vector-valued distributions, which we recall here for completeness. In order to define weak derivatives consider $D((0, T))$, the space of real valued $C^{\infty}$ smooth functions with compact support in $(0, T)$. Fix $s \in[0, T]$. Each $y \in L^{2}\left((0, T), H^{1}(\Gamma(s))\right)$ 
defines a vector-valued distribution $\mathcal{J}_{y}: Ð((0, T)) \rightarrow H^{1}(\Gamma(s))$ through the $H^{1}(\Gamma(s))$-valued integral $\int_{[0, T]} y(t) \varphi(t) \mathrm{d} t$.

Its distributional derivative is said to lie in $L^{2}\left((0, T), H^{-1}(\Gamma(s))\right)$ if it can be represented by $w \in L^{2}\left((0, T), H^{-1}(\Gamma(s))\right)$ in the following sense

$$
\forall \varphi \in \boxplus\left((0, T), H^{1}(\Gamma(s))\right): \int_{[0, T]}\left\langle y(t), \varphi^{\prime}(t)\right\rangle_{L^{2}(\Gamma(s))}+\langle w(t), \varphi(t)\rangle_{H^{-1}(\Gamma(s)), H^{1}(\Gamma(s))} \mathrm{d} t=0,
$$

and we write $y^{\prime}=w$. Here and in the following, by $H^{-1}$ we denote the representation of the dual $\left(H^{1}\right)^{*}$ which arises from $L^{2} \supset H^{1}$ by completion.

Let us summarize the definition and some well known properties of the space $W(0, T)$, compare [17, Ch. I, Theorems 3.1 and 2.1], [10, Ch. 5, Theorem 3], and [24, Theorem 3.10].

LEMMA 2.2 For $s \in[0, T]$, the space

$$
W_{s}(0, T)=\left\{v \in L^{2}\left((0, T), H^{1}(\Gamma(s))\right) \mid v^{\prime} \in L^{2}\left((0, T), H^{-1}(\Gamma(s))\right)\right\}
$$

with scalar product $\int_{0}^{T}\langle\cdot, \cdot\rangle_{H^{1}(\Gamma(s))}+\left\langle(\cdot)^{\prime},(\cdot)^{\prime}\right\rangle_{H^{-1}(\Gamma(s))} \mathrm{d} t$ is a Hilbert space.

1. $W_{s}(0, T)$ is compactly embedded into $C\left([0, T], L^{2}(\Gamma(s))\right)$, the space of continuous $L^{2}$-valued functions.

2. Denote by $\Phi\left([0, T], H^{1}(\Gamma(s))\right)$ the space of $C^{\infty}$-smooth $H^{1}(\Gamma(s))$-valued test functions on $[0, T]$. The inclusion $Ð\left([0, T], H^{1}(\Gamma(s))\right) \subset W_{s}(0, T)$ is dense.

3. For two functions $v, w \in W_{s}(0, T)$ the product $\langle v(t), w(t)\rangle_{L^{2}(\Gamma(s))}$ is absolutely continuous with respect to $t \in[0, T]$ and

$$
\frac{\mathrm{d}}{\mathrm{d} t} \int_{\Gamma(s)} v(t) w(t) \mathrm{d} \Gamma(s)=\left\langle v^{\prime}, w\right\rangle_{H^{-1}(\Gamma(s)), H^{1}(\Gamma(s))}+\left\langle v, w^{\prime}\right\rangle_{H^{1}(\Gamma(s)), H^{-1}(\Gamma(s))},
$$

a.e. in $(0, T)$, and as a consequence there holds the formula of integration by parts

$$
\int_{[r, t]}\left\langle v^{\prime}, w\right\rangle_{H^{-1}, H^{1}} \mathrm{~d} \tau=\langle v(t), w(t)\rangle_{L^{2}(\Gamma(s))}-\langle v(r), w(r)\rangle_{L^{2}(\Gamma(s))}-\int_{[r, t]}\left\langle v, w^{\prime}\right\rangle_{H^{1}, H^{-1}} \mathrm{~d} \tau .
$$

\section{Weak solutions}

The scope of this section is to formulate appropriate function spaces and a related weak material derivative, in order to prove the existence of unique weak solutions of (1.1) for quite weak right-hand sides $f$.

We start by defining the strong material derivative for smooth functions $f \in C^{1}\left(\mathbb{R}^{n+1} \times[0, T]\right)$, namely the derivative

$$
\dot{f}(x, t)=\left.\frac{\mathrm{d}}{\mathrm{d} s}\right|_{s=t} f\left(\bar{\Phi}_{s}^{t}(x), s\right)=\nabla f(x, t) V(x, t)+\partial_{t} f(x, t),
$$

along trajectories of the velocity field $V$. The material derivative has the following properties. 
LEMMA 3.1 Let $f$ be sufficiently smooth. Then

$$
\frac{\mathrm{d}}{\mathrm{d} t} \int_{\Gamma(t)} f \mathrm{~d} \Gamma(t)=\int_{\Gamma(t)} \dot{f}+f \operatorname{div} \Gamma V \mathrm{~d} \Gamma(t)
$$

and

$$
\frac{\mathrm{d}}{\mathrm{d} t} \int_{\Gamma(t)}\left\|\nabla_{\Gamma} f\right\|^{2} \mathrm{~d} \Gamma(t)=\int_{\Gamma(t)} 2 \nabla_{\Gamma} f \cdot \nabla_{\Gamma} \dot{f}-2 \nabla_{\Gamma} f\left(D_{\Gamma} V\right) \nabla_{\Gamma} f+\left\|\nabla_{\Gamma} f\right\|^{2} \operatorname{div}{ }_{\Gamma} V \mathrm{~d} \Gamma(t),
$$

with $\operatorname{div}{ }_{\Gamma(t)} V=\sum_{i=1}^{n+1} \nabla_{\Gamma(t)}^{i} V^{i}$ and $\left(D_{\Gamma(t)} V\right)_{i j}=\nabla_{\Gamma(t)}^{j} V^{i}$.

A proof and details can be found in the Appendix of [3].

LEMmA 3.2 Let $J_{t}^{s}(\cdot)=\operatorname{det} \mathrm{D}_{\Gamma(s)} \Phi_{t}^{s}(\cdot)$ denote the Jacobian determinant of the matrix representation of $\mathrm{D}_{\Gamma(s)} \Phi_{t}^{s}(\cdot)$ with respect to orthogonal bases of the respective tangent space.

By Assumption $2.1 J_{t}^{s} \in C^{1}([0, T] \times \Gamma(s))$ and there exists $C_{J}>0$, such that for all $s, t \in[0, T]$

$$
\frac{1}{C_{J}} \leqslant \min _{\gamma \in \Gamma(s)} J_{t}^{s}(\gamma) \leqslant \max _{\gamma \in \Gamma(s)} J_{t}^{s}(\gamma) \leqslant C_{J} .
$$

Given Assumption 2.1, consider the family $\left\{L^{2}(\Gamma(t))\right\}_{t \in[0, T]}$. Then for $v \in L^{2}(\Gamma(t))$ we introduce the pull-back

$$
\phi_{t}^{s} v=v\left(\Phi_{t}^{s}(\cdot)\right) \in L^{2}(\Gamma(s)),
$$

which is a linear homeomorphism from $L^{2}(\Gamma(t))$ into $L^{2}(\Gamma(s))$ for any $s, t \in[0, T]$. Moreover $\phi_{t}^{s}$ is a linear homeomorphism from $H^{1}(\Gamma(t))$ into $H^{1}(\Gamma(s))$. Thus finally the adjoint operator, $\phi_{t}^{s *}$ : $H^{-1}(\Gamma(s)) \rightarrow H^{-1}(\Gamma(t))$ is also a linear homeomorphism. There exist constants $C_{L^{2}(\Gamma)}, C_{H^{1}(\Gamma)}$ independent of $s, t$, such that for all $v \in L^{2}(\Gamma(t))$, or $v \in H^{1}(\Gamma(t))$ respectively, and for all $s, t \in[0, T]$

$$
\left\|\phi_{t}^{s} v\right\|_{H^{1}(\Gamma(s))} \leqslant C_{H^{1}(\Gamma)}\|v\|_{H^{1}(\Gamma(t))}, \quad\left\|\phi_{t}^{s} v\right\|_{L^{2}(\Gamma(s))} \leqslant C_{L^{2}(\Gamma)}\|v\|_{L^{2}(\Gamma(t))},
$$

and thus finally $\left\|\phi_{t}^{s^{*}}\right\|_{\mathcal{L}\left(H^{-1}(\Gamma(s)), H^{-1}(\Gamma(t))\right)} \leqslant C_{H^{1}(\Gamma)}$.

Furthermore there holds $\partial_{t} J_{t}^{S}=\phi_{t}^{s}\left(\operatorname{div}_{\Gamma(t)} V\right) J_{t}^{S}$.

Proof. The proof of equivalence of the $H^{1}$ - and the $L^{2}$-norms follows the lines of, e.g., of [7, Ch. 9 , SubSec. 4.1]. Now because $\|\cdot\|_{H^{1}(\Gamma(t))}$ and $\left\|\phi_{t}^{s}(\cdot)\right\|_{H^{1}(\Gamma(s))}$ are two equivalent norms on $H^{1}(\Gamma(t))$ also their dual norms are equivalent, as shows the following short argument. The dual norm of a functional from the dual space $v^{*} \in\left(H^{1}(\Gamma(s))\right)^{*}$ can now be expressed by

$$
\sup _{w \in H^{1}(\Gamma(s))} \frac{\left\langle v^{*}, w\right\rangle_{\left(H^{1}(\Gamma(s))\right)^{\prime}, H^{1}(\Gamma(s))}}{\|w\|_{H^{1}(\Gamma(s))}}=\sup _{v \in H^{1}(\Gamma(t))} \frac{\left\langle\phi_{t}^{s *} v^{*}, v\right\rangle_{\left(H^{1}(\Gamma(t))\right)^{\prime}, H^{1}(\Gamma(t))}}{\left\|\phi_{t}^{S} v\right\|_{H^{1}(\Gamma(s))}},
$$

and the bound on the norm of $\phi_{t}^{s *}$ follows from the equivalence of said $H^{1}$-norms.

The last assertion is a by-product of the proof of Lemma 3.1, compare [3].

We need to state one more Lemma concerning continuous time-dependence of the previously defined norms. 
Lemma 3.3 Let $s \in[0, T]$. For $v_{1} \in H^{1}(\Gamma(s)), v_{2} \in L^{2}(\Gamma(s)), v_{3} \in H^{-1}(\Gamma(s))$ the following expressions are continuous with respect to $t \in[0, T]$

$$
\left\|\phi_{s}^{t} v_{1}\right\|_{H^{1}(\Gamma(t))}, \quad\left\|\phi_{s}^{t} v_{2}\right\|_{L^{2}(\Gamma(t))}, \quad\left\|\phi_{t}^{s^{*}} v_{3}\right\|_{H^{-1}(\Gamma(t))} .
$$

Proof. For the first two norms this is a standard task of shape calculus, compare, e.g., [7, Ch. 8, Section 4.3.2]. By the change of variables formula we have

$$
\left\|\phi_{s}^{t} v_{1}\right\|_{H^{1}(\Gamma(t))}^{2}=\int_{\Gamma(s)}\left(\nabla_{\Gamma} v_{1}\left(D_{\Gamma(s)} \bar{\Phi}_{t}^{s}\right)^{-1}\left(D_{\Gamma(s)} \bar{\Phi}_{t}^{s}\right)^{-T} \nabla_{\Gamma} v_{1}+v_{1}^{2}\right) J_{t}^{s} \mathrm{~d} \Gamma(s),
$$

which is a continuous function due to the regularity of $\Phi$ stated in Assumption 2.1. Omit the term involving the gradient in (3.3) and the same argument proves continuity of the $L^{2}$-norm.

Moreover, since on the tangential space $T \Gamma(s)$ we have $\left(D_{\Gamma(s)} \bar{\Phi}_{s}^{s}\right)^{-1}\left(D_{\Gamma(s)} \bar{\Phi}_{s}^{s}\right)^{-T}=\mathrm{id}_{T \Gamma(s)}$, and since there holds $J_{s}^{s}=1$ and $\Phi_{(\cdot)}^{s}(\cdot) \in C^{2}\left(\Gamma(s) \times[0, T], \mathbb{R}^{n+1}\right)$ Equation (3.3) yields

$$
\left|\left\|\phi_{s}^{t} v\right\|_{H^{1}(\Gamma(t))}^{2}-\|v\|_{H^{1}(\Gamma(s))}^{2}\right| \leqslant C|t-s|\|v\|_{H^{1}(\Gamma(s))}^{2},
$$

for all $v \in H^{1}(\Gamma(s))$. Regarding (3.2) this allows us to estimate

$$
\frac{1}{(1+C|s-t|)^{\frac{1}{2}}}\left\|v_{3}\right\|_{H^{-1}(\Gamma(s))} \leqslant\left\|\phi_{t}^{s *} v_{3}\right\|_{H^{-1}(\Gamma(t))} \leqslant \frac{1}{(1-C|s-t|)^{\frac{1}{2}}}\left\|v_{3}\right\|_{H^{-1}(\Gamma(s))} .
$$

As far as Lemma 3.1 is concerned, for a family of functions $\{f(t)\}_{t \in[0, T]}, f(t): \Gamma(t) \rightarrow \mathbb{R}$, one can define $\dot{f}$ at $\gamma=\Phi_{t}^{0} \gamma_{0}$ simply by $\dot{f}(t)[\gamma]=\phi_{0}^{t} \frac{\mathrm{d}}{\mathrm{d} t}\left(\phi_{t}^{0} f(t)\right)\left[\gamma_{0}, t\right]=\phi_{0}^{t} \frac{\mathrm{d}}{\mathrm{d} t}\left[f(t)\left(\Phi_{t}^{0} \gamma_{0}\right)\right]$. If $\{f(t)\}$ can be smoothly extended, this is equivalent to (3.1). The following Lemmas aim at defining a weak material derivative of $f$ that translates into a weak derivative of the pull-back $\phi_{t}^{0} f(t)$.

Definition 3.4 Consider the disjoint union $\mathbb{B}_{L^{2}}=\bigcup_{t \in[0, T]} L^{2}(\Gamma(t)) \times\{t\}$. The set of sections $f:[0, T] \rightarrow \mathbb{B}_{L^{2}}, t \mapsto(v, t)$ inherits a canonical vector space structure from the spaces $L^{2}(\Gamma(t))$ (addition and multiplications with scalars). Given Assumption 2.1, for $s \in[0, T]$ consider the space

$$
L_{L^{2}(\Gamma)}^{2}:=\left\{\bar{v}:[0, T] \rightarrow \mathbb{B}_{L^{2}}, t \mapsto\left(v_{t}, t\right) \mid \phi_{t}^{s} v \in L^{2}\left((0, T), L^{2}(\Gamma(s))\right)\right\} .
$$

Abusing notation, now and in the following we identify $\bar{v}(t)=\left(v_{t}, t\right) \in L_{L^{2}(\Gamma)}^{2}$ with $v(t)=v_{t}$.

In the same manner we define the space $L_{H^{1}(\Gamma)}^{2}$. For $L_{H^{-1}(\Gamma)}^{2}$ use $\phi_{s}^{t *}$ instead of $\phi_{t}^{s}$.

For $\varphi \in \phi_{s}^{(\cdot)} D\left((0, T), H^{1}(\Gamma(s))\right)=\left\{\varphi \in L_{L^{2}(\Gamma)}^{2} \mid \phi_{t}^{s} \varphi \in \boxplus\left((0, T), H^{1}(\Gamma(s))\right\}\right.$, it is clear how to interpret $\dot{\varphi}$, namely $\dot{\varphi}=\phi_{s}^{t}\left(\phi_{t}^{s} \varphi\right)^{\prime} \in H^{1}(\Gamma(t))$. We say that $y \in L_{H^{1}(\Gamma)}^{2}$ has weak material derivative $\dot{y}(t) \in L_{H^{-1}(\Gamma)}^{2}$ iff there holds

$$
\int_{[0, T]}\langle\dot{y}, \varphi\rangle_{H^{-1}(\Gamma(t)), H^{1}(\Gamma(t))} \mathrm{d} t=-\int_{[0, T]}\langle y, \dot{\varphi}\rangle_{L^{2}(\Gamma(t))} \mathrm{d} t-\int_{[0, T] \Gamma(t)} \int_{\Gamma(t)} y \varphi \operatorname{div} \Gamma V \mathrm{~d} \Gamma(t) \mathrm{d} t
$$

for all $\varphi \in \phi_{s}^{(\cdot)} \boxplus\left((0, T), H^{1}(\Gamma(s))\right)$. 
LEMMA 3.5 Endowed with the scalar product

$$
\langle f, g\rangle_{L_{L^{2}(\Gamma)}^{2}}=\int_{[0, T]}\langle f(t), g(t)\rangle_{L^{2}(\Gamma(t))} \mathrm{d} t
$$

$L_{L^{2}(\Gamma)}^{2}$ is a Hilbert space. Analogously one can define scalar products on $L_{H^{1}(\Gamma)}^{2}$ and $L_{H^{-1}(\Gamma)}^{2}$. All three spaces do not depend on $s$. Also the definition of the weak material derivative $\dot{y}$ from 3.4 does not depend on $s$.

Proof. In order to define the scalar product of $L_{L^{2}(\Gamma)}^{2}$, we must ensure measurability of $\langle f, g\rangle_{L^{2}(\Gamma(t))}:[0, T] \rightarrow \mathbb{R}$. Since $\langle f, g\rangle=\frac{1}{2}\left(\|f+g\|^{2}-\|f\|^{2}-\|g\|^{2}\right)$ it suffices to show measurability of $\|f\|_{L^{2}(\Gamma(t))}^{2}$ for all $f \in L_{L^{2}(\Gamma)}^{2}$. By definition of the set $L_{L^{2}(\Gamma)}^{2}$ we have $\phi_{t}^{s} f \in L^{2}\left([0, T], L^{2}(\Gamma(s))\right)$. Hence, there exists a sequence of measurable simple functions $\tilde{f}_{n}$ that converge pointwise a.e. to $\phi_{t}^{s} f$ in $L^{2}(\Gamma(s))$. Each $\tilde{f}_{n}$ is the finite sum of measurable singlevalued functions, i.e. $\tilde{f}_{n}=\sum_{i=1}^{M_{n}} f_{i, n} \mathbf{1}_{B_{i}}, M_{n} \in \mathbb{N}, f_{i, n} \in L^{2}(\Gamma(s))$, [0,T] $\supset B_{i}$ measurable and disjoint. By Lemma 3.3 the function

$$
\left\|\phi_{s}^{t} \tilde{f}_{n}\right\|_{L^{2}(\Gamma(t))}=\sum_{i=1}^{M_{n}}\left\|\phi_{s}^{t} f_{i, n}\right\|_{L^{2}(\Gamma(t))} \mathbf{1}_{B_{i}}
$$

is the finite sum of measurable functions and thus measurable. Using the continuity of the operator $\phi_{s}^{t}$, as stated in Lemma 3.2, one infers pointwise convergence a.e. of $\left\|\phi_{s}^{t} \tilde{f}_{n}\right\|_{L^{2}(\Gamma(t))}$ towards $\|f\|_{L^{2}(\Gamma(t))}$ which in turn implies measurability of $\|f\|_{L^{2}(\Gamma(t))}$.

Again by Lemma 3.2 we now conclude integrability of $\|f\|_{L^{2}(\Gamma(t))}$ and at the same time equivalence of the norms

$$
\left(\int_{[0, T]}\|f\|_{L^{2}(\Gamma(t))}^{2} \mathrm{~d} t\right)^{\frac{1}{2}} \text { and }\left(\int_{[0, T]}\left\|\phi_{t}^{S} f\right\|_{L^{2}(\Gamma(s))}^{2} \mathrm{~d} t\right)^{\frac{1}{2}}
$$

Completeness of $L_{L^{2}(\Gamma)}^{2}$ follows, since $L_{L^{2}(\Gamma)}^{2}$ and $L^{2}\left((0, T), L^{2}(\Gamma(s))\right)$ are isomorph. Again because of Lemma 3.2, $\phi_{t}^{s} v \in L^{2}\left((0, T), L^{2}(\Gamma(s))\right)$ is equivalent to $\phi_{t}^{r} v \in L^{2}\left((0, T), L^{2}(\Gamma(r))\right)$, thus the definition does not depend on the choice of $s$. For $L_{H^{1}(\Gamma)}^{2}$ and $L_{H^{-1}(\Gamma)}^{2}$ we proceed similarly.

We show that the definition of the weak material derivative does not depend on $s \in[0, T]$. On $\Gamma(s)$ Equation (3.4) reads

$$
\int_{[0, T]}\left\langle\phi_{s}^{t^{*}} \dot{y}, \tilde{\varphi}\right\rangle_{H^{-1}(\Gamma(s)), H^{1}(\Gamma(s))} \mathrm{d} t=-\int_{[0, T]} \int_{\Gamma(s)}\left(\phi_{t}^{s} y \tilde{\varphi}^{\prime}(t)+\phi_{t}^{s}(y \operatorname{div} \Gamma(t) V) \tilde{\varphi}\right) J_{t}^{s} \mathrm{~d} \Gamma(s) \mathrm{d} t
$$

for all $\tilde{\varphi} \in \nsupseteq\left([0, T], H^{1}(\Gamma(s))\right)$. For $r \in[0, T]$, we now transform the relation into one on $\Gamma(r)$, 
using $\phi_{s}^{r},\left(\phi_{r}^{S}\right)^{*}$ and $\phi_{t}^{r}=\phi_{s}^{r} \circ \phi_{t}^{s}$

$$
\begin{aligned}
\int_{[0, T]}\left\langle\phi_{r}^{t^{*}} \dot{y}, \phi_{s}^{r} \tilde{\varphi}\right\rangle_{H^{-1}(\Gamma(r)), H^{1}(\Gamma(r))} \mathrm{d} t & \\
= & -\int_{[0, T]} \int_{\Gamma(r)}\left(\phi_{t}^{r} y\left(\phi_{s}^{r} \tilde{\varphi}(t)\right)^{\prime}+\phi_{t}^{r}\left(y \operatorname{div} \Gamma_{(t)} V\right) \phi_{s}^{r} \tilde{\varphi}\right) J_{t}^{r} \mathrm{~d} \Gamma(r) \mathrm{d} t,
\end{aligned}
$$

and because $\phi_{s}^{r}: H^{1}(\Gamma(s)) \rightarrow H^{1}(\Gamma(r))$ is a linear homeomorphism, it also defines an isomorphism between $D\left([0, T], H^{1}(\Gamma(s))\right)$ and $D\left([0, T], H^{1}(\Gamma(r))\right)$.

REMARK 3.6 Strictly speaking the elements of $L_{X(\Gamma)}^{2}$ are equivalence classes of functions coinciding a.e. in $[0, T]$, just like the elements of $L^{2}((0, T), X(\Gamma(s)))$.

The definition of the weak derivative of $y \in L_{H^{1}(\Gamma)}^{2}$ in (3.4) translates into weak derivatives of the pullback $\phi_{t}^{s} y$. In order to make the connection between the two, we state the following

Lemma 3.7 Let $w \in W_{s}(0, T)$ and $f \in C^{1}([0, T] \times \Gamma(s))$. Then $f w$ also lies in $W_{s}(0, T)$ and

$$
(f w)^{\prime}=\underbrace{\partial_{t} f w}_{\in L^{2}\left([0, T], L^{2}(\Gamma(s))\right)}+f w^{\prime},
$$

where $f w^{\prime}$ is to be understood as $\left\langle f w^{\prime}, \varphi\right\rangle_{H^{-1}(\Gamma(s)), H^{1}(\Gamma(s))}=\left\langle w^{\prime}, f \varphi\right\rangle_{H^{-1}(\Gamma(s)), H^{1}(\Gamma(s))}$.

Proof. Making use of the uniform continuity of $f$ on the compact tube $[0, T] \times \Gamma(s)$, one can show that for $\varphi \in \Phi\left((0, T), H^{1}(\Gamma(s))\right)$ the function $f \varphi$ lies in $W_{s}(0, T)$ and that $(f \varphi)^{\prime}=f^{\prime} \varphi+f \varphi^{\prime}$. The claim then follows by integration by parts in $W_{s}(0, T)$ as

$$
\begin{aligned}
\int_{[0, T]}\left\langle w^{\prime}, f \varphi\right\rangle_{H^{-1}(\Gamma(s)), H^{1}(\Gamma(s))} \mathrm{d} t & =-\int_{[0, T]}\left\langle w,(f \varphi)^{\prime}\right\rangle_{H^{1}(\Gamma(s)), H^{-1}(\Gamma(s))} \mathrm{d} t \\
& =-\int_{[0, T]}\left\langle w, \partial_{t} f \varphi\right\rangle_{L^{2}(\Gamma(s))} \mathrm{d} t-\int_{[0, T]}\left\langle w, f \varphi^{\prime}\right\rangle_{L^{2}(\Gamma(s))} \mathrm{d} t .
\end{aligned}
$$

Reordering gives

$$
\int_{[0, T]}\left\langle f w, \varphi^{\prime}\right\rangle_{L^{2}(\Gamma(s))} \mathrm{d} t=-\int_{[0, T]}\left\langle\partial_{t} f w+f w^{\prime}, \varphi\right\rangle_{H^{-1}(\Gamma(s)), H^{1}(\Gamma(s))} \mathrm{d} t
$$

for any $\varphi \in \oiint\left((0, T), H^{1}(\Gamma(s))\right)$. Hence condition (2.2) holds for $f w$. Using the density property stated in Lemma 2.2[2.], we can approximate $f w$ by continuous $H^{1}(\Gamma(s))$-valued functions and infer $f w \in L^{2}\left((0, T), H^{1}(\Gamma(s))\right)$. The same argument yields $\partial_{t} f w+f w^{\prime} \in$ $L^{2}\left((0, T), H^{-1}(\Gamma(s))\right)$.

Finally we can define our solution space of (1.1).

DEFINITION 3.8 The solution space $W_{\Gamma}$ is defined as follows

$$
W_{\Gamma}=\left\{v \in L_{H^{1}(\Gamma)}^{2} \mid \dot{v} \in L_{H^{-1}(\Gamma)}^{2}\right\} .
$$


LEMMA $3.9 W_{\Gamma}$ is Hilbert with the canonical scalar product $\int_{0}^{T}\langle\cdot, \cdot\rangle_{H^{1}(\Gamma(t))}+\langle(\dot{\cdot}),(\cdot \cdot)\rangle_{H^{-1}(\Gamma(t))} \mathrm{d} t$. Also $y \in W_{\Gamma}$ iff $\phi_{t}^{s} y \in W_{s}(0, T)$ for (every) $s \in[0, T]$. For all $\tilde{\varphi} \in \Phi\left((0, T), H^{1}(\Gamma(s))\right)$ there holds

$$
\int_{[0, T]}\left\langle\phi_{s}^{t^{*}} \dot{y}, \tilde{\varphi}\right\rangle_{H^{-1}(\Gamma(s)), H^{1}(\Gamma(s))} \mathrm{d} t=\int_{[0, T]}\left\langle\left(\left(\phi_{t}^{s} y\right)^{\prime}, J_{t}^{s} \tilde{\varphi}\right\rangle_{H^{-1}(\Gamma(s)), H^{1}(\Gamma(s))} \mathrm{d} t .\right.
$$

One has

$$
c_{W}\left\|\phi_{t}^{s} y\right\|_{W_{s}(0, T)} \leqslant\|y\|_{W_{\Gamma}} \leqslant C_{W}\left\|\phi_{t}^{s} y\right\|_{W_{s}(0, T)},
$$

and $c_{W}, C_{W}>0$ do not depend on $s \in[0, T]$.

Proof. For $y \in W_{\Gamma}$, observe that $J_{t}^{s} \phi_{t}^{s} y \in L^{2}\left([0, T], H^{1}(\Gamma(s))\right.$ and rewrite (3.5) as

$$
\begin{array}{r}
\int_{[0, T]}\left\langle J_{t}^{s} \phi_{t}^{s} y, \partial_{t} \tilde{\varphi}\right\rangle_{L^{2}(\Gamma(s))} \mathrm{d} t=-\int_{[0, T]}\left\langle\phi_{s}^{t *} \dot{y}, \tilde{\varphi}\right\rangle_{H^{-1}(\Gamma(s)), H^{1}(\Gamma(s))} \mathrm{d} t \\
-\int_{[0, T]}\left\langle\partial_{t} J_{t}^{s} \phi_{t}^{s} y, \tilde{\varphi}\right\rangle_{L^{2}(\Gamma(s))} \mathrm{d} t,
\end{array}
$$

for $\tilde{\varphi} \in \Phi\left((0, T), H^{1}(\Gamma(s))\right)$. Hence $J_{t}^{s} \phi_{t}^{s} y \in W_{s}(0, T)$, and from Lemma 3.7 it follows that also $\phi_{t}^{s} y \in W_{s}(0, T)$, because $\frac{1}{J_{t}^{s}} \in C^{1}([0, T] \times \Gamma(s))$. Note that we used $\partial_{t} J_{t}^{s}=\phi_{t}^{s}(\operatorname{div} \Gamma(t) V) J_{t}^{s}$, see Lemma 3.2. On the other hand, for any $\tilde{y} \in W_{s}(0, T)$ one has $J_{t}^{s} \tilde{y} \in W_{s}(0, T)$ and thus $y=\phi_{s}^{t} \tilde{y} \in W_{\Gamma}$. Hence $\phi_{(\cdot)}^{s}$ constitutes an isomorphism between $W_{\Gamma}$ and $W_{s}(0, T)$.

Apply Lemma 3.7 a second time to obtain $\left(J_{t}^{s} \tilde{\varphi}\right)^{\prime}=\partial_{t} J_{t}^{s} \tilde{\varphi}+J_{t}^{s} \tilde{\varphi}^{\prime}$ and because of $\tilde{\varphi}(0)=$ $\tilde{\varphi}(T)=0 \in H^{1}(\Gamma(s))$ by integration by parts there follows from (3.7)

$$
\int_{[0, T]}\left\langle\phi_{s}^{t *} \dot{y}, \tilde{\varphi}\right\rangle_{H^{-1}(\Gamma(s)), H^{1}(\Gamma(s))} \mathrm{d} t=\int_{[0, T]}\left\langle\left(\left(\phi_{t}^{s} y\right)^{\prime}, J_{t}^{s} \tilde{\varphi}\right\rangle_{H^{-1}(\Gamma(s)), H^{1}(\Gamma(s))} \mathrm{d} t,\right.
$$

compare Lemma 2.2[3.]. This proves the second claim.

The claim of $W_{\Gamma}$ being Hilbert now follows. Observe that point-wise multiplication with $J_{t}^{s}$ constitutes a linear homeomorphism in $H^{1}(\Gamma(s))$ whose inverse is the multiplication by $\frac{1}{J_{t}^{s}}$. One easily checks $\left\|J_{t}^{S} \varphi\right\|_{H^{1}(\Gamma(s))} \leqslant c\left\|J_{t}^{S}\right\|_{C^{1}(\Gamma(s))}\|\varphi\|_{H^{1}(\Gamma(s))} \leqslant C\|\varphi\|_{H^{1}(\Gamma(s))}$. This together with Lemma 3.2 yields the equivalence of the two norms on $W_{\Gamma}$

$$
\int_{[0, T]}\|y\|_{H^{1}(\Gamma(t))}^{2}+\|\dot{y}\|_{H^{-1}(\Gamma(t))}^{2} \mathrm{~d} t \quad \text { and } \int_{[0, T]}\left\|\phi_{t}^{s} y\right\|_{H^{1}(\Gamma(s))}^{2}+\left\|\left(\phi_{t}^{s} y\right)^{\prime}\right\|_{H^{-1}(\Gamma(s))}^{2} \mathrm{~d} t .
$$

Completeness of $W_{s}(0, T)$ then implies completeness of $W_{\Gamma}$.

REMARK 3.10 Formula (3.6) can be seen as a generalization of the following relation. Assume $\phi_{t}^{s} y \in Ð\left((0, T), H^{1}(\Gamma(s))\right)$. Then

$$
\int_{[0, T]}\left\langle\phi_{s}^{t *} \dot{y}, \tilde{\varphi}\right\rangle_{H^{-1}(\Gamma(s)), H^{1}(\Gamma(s))} \mathrm{d} t=\int_{[0, T]}\left\langle\dot{y}, \phi_{s}^{t} \tilde{\varphi}\right\rangle_{L^{2}(\Gamma(t))} \mathrm{d} t=\int_{[0, T]}\left\langle\left(\phi_{t}^{s} y\right)^{\prime}, J_{t}^{s} \tilde{\varphi}\right\rangle_{L^{2}(\Gamma(s))} \mathrm{d} t .
$$


Using Lemma 3.7 and 2.2, it is now easy to prove

Lemma 3.11 For two functions $v, w \in W_{\Gamma}$ the expression $\langle v(t), w(t)\rangle_{L^{2}(\Gamma(t))}$ is absolutely continuous with respect to $t \in[0, T]$ and

$$
\begin{aligned}
\frac{1}{\mathrm{~d} t} \int_{\Gamma(t)} v w \mathrm{~d} \Gamma(t)=\langle\dot{v}, w\rangle_{H^{-1}(\Gamma(t)), H^{1}(\Gamma(t))} & \\
& +\langle v, \dot{w}\rangle_{H^{1}(\Gamma(t)), H^{-1}(\Gamma(t))}+\int_{\Gamma(t)} v w \operatorname{div} \Gamma(t) V \mathrm{~d} \Gamma(t),
\end{aligned}
$$

a.e. in $(0, T)$, and there holds the formula of integration by parts

$$
\begin{aligned}
\int_{[s, t]}\langle\dot{v}, w\rangle_{H^{-1}(\Gamma(\tau)), H^{1}(\Gamma(\tau))} \mathrm{d} \tau & =\langle v, w\rangle_{L^{2}(\Gamma(t))}-\langle v, w\rangle_{L^{2}(\Gamma(s))} \\
& -\int_{[s, t]}\left[\langle v, \dot{w}\rangle_{H^{1}(\Gamma(\tau)), H^{-1}(\Gamma(\tau))}+\int_{\Gamma(\tau)} v w \operatorname{div}_{\Gamma} V \mathrm{~d} \Gamma(\tau)\right] \mathrm{d} \tau .
\end{aligned}
$$

We can now formulate (1.1) in a weak and slightly generalized manner. Let $\tilde{b} \in C^{1}\left([0, T] \times \Gamma_{0}\right)$ and $b=\phi_{0}^{t} \tilde{b}$. We look for solutions $u \in W_{\Gamma}$ that satisfy $y(0)=y_{0} \in L^{2}\left(\Gamma_{0}\right)$ and for $f \in L_{H^{-1}(\Gamma)}^{2}$

$$
\begin{aligned}
\frac{\mathrm{d}}{\mathrm{d} t} \int_{\Gamma(t)} y \varphi \mathrm{d} \Gamma(t)+\int_{\Gamma(t)} \nabla_{\Gamma} y \cdot \nabla_{\Gamma} \varphi+b y \varphi \mathrm{d} \Gamma(t)=\langle\dot{\varphi}, y\rangle_{H^{-1}(\Gamma(t)), H^{1}(\Gamma(t))} & \\
& +\langle f, \varphi\rangle_{H^{-1}(\Gamma(t)), H^{1}(\Gamma(t))},
\end{aligned}
$$

for all $\varphi \in W_{\Gamma}$ and a.e. $t \in(0, T)$. One may equivalently write (3.8) as

$$
\dot{y}+\Delta_{\Gamma(t)} y+y\left(\operatorname{div}_{\Gamma(t)} V+b\right)=f \quad \text { in } H^{-1}(\Gamma(t))
$$

for a.e. $t \in(0, T)$. We apply known existence and uniqueness results for the pulled-back equation to prove

THEOREM 3.12 Let $f \in L_{H^{-1}(\Gamma)}^{2}, y_{0} \in L^{2}\left(\Gamma_{0}\right)$. There exists a unique $y \in W_{\Gamma}$, such that (3.8) is fulfilled for all $\phi \in W_{\Gamma}$ and a.e. $t \in(0, T)$. There holds

$$
\|y\|_{W_{\Gamma}} \leqslant C\left(\left\|y_{0}\right\|_{L^{2}\left(\Gamma_{0}\right)}+\|f\|_{L_{H^{-1}(\Gamma)}^{2}}\right) .
$$

Proof. Let us relate equation (3.8) to the fixed domain $\Gamma(s)$ via

$$
\begin{aligned}
\frac{\mathrm{d}}{\mathrm{d} t} \int_{\Gamma(s)} \tilde{y} \tilde{\varphi} J_{t}^{s} \mathrm{~d} \Gamma(s)+\int_{\Gamma(s)}\left(\nabla_{\Gamma} \tilde{y}\left(D_{\Gamma(s)} \bar{\Phi}_{t}^{s}\right)^{-1}\left(D_{\Gamma(s)} \bar{\Phi}_{t}^{s}\right)^{-T} \nabla_{\Gamma} \tilde{\varphi}+\tilde{b} \tilde{y} \tilde{\varphi}\right) J_{t}^{s} \mathrm{~d} \Gamma(s) \\
\quad=\left\langle\tilde{\varphi}^{\prime}, J_{t}^{s} \tilde{y}\right\rangle_{H^{-1}(\Gamma(s)), H^{1}(\Gamma(s))}+\left\langle\tilde{f}, J_{t}^{s} \tilde{\varphi}\right\rangle_{H^{-1}(\Gamma(s)), H^{1}(\Gamma(s))},
\end{aligned}
$$


with $\tilde{y}=\phi_{t}^{s} y, \tilde{f}=\frac{1}{J_{t}^{s}} \phi_{s}^{t *} f \in L^{2}\left((0, T), H^{-1}(\Gamma(s))\right.$ and for all $\phi_{t}^{s} \varphi=\tilde{\varphi} \in W_{s}(0, T)$. This again is equivalent to

$$
\begin{aligned}
& \left\langle\tilde{y}^{\prime}, \tilde{\varphi} J_{t}^{s}\right\rangle_{H^{-1}(\Gamma(s)), H^{1}(\Gamma(s))}+\int_{\Gamma(s)} \tilde{y} \tilde{\varphi}\left(\phi_{t}^{s}\left(\operatorname{div}_{\Gamma(t)} V\right)+\tilde{b}\right) J_{t}^{s} \mathrm{~d} \Gamma(s) \\
& \quad+\int_{\Gamma(s)} \nabla_{\Gamma} \tilde{y}\left(D_{\Gamma(s)} \bar{\Phi}_{t}^{s}\right)^{-1}\left(D_{\Gamma(s)} \bar{\Phi}_{t}^{s}\right)^{-T} \nabla_{\Gamma} \tilde{\varphi} J_{t}^{s} \mathrm{~d} \Gamma(s)=\left\langle\tilde{f}, J_{t}^{s} \tilde{\varphi}\right\rangle_{H^{-1}(\Gamma(s)), H^{1}(\Gamma(s))}
\end{aligned}
$$

With $\psi=J_{t}^{s} \tilde{\varphi}$ one gets for all $\psi \in W_{s}(0, T)$

$$
\left\langle\tilde{y}^{\prime}, \psi\right\rangle_{H^{-1}(\Gamma(s)), H^{1}(\Gamma(s))}+a(t, \tilde{y}, \psi)=\langle\tilde{f}, \psi\rangle_{H^{-1}(\Gamma(s)), H^{1}(\Gamma(s))},
$$

with a bilinear form

$$
\begin{gathered}
a(t, \tilde{y}, \psi)=\int_{\Gamma(s)} \nabla_{\Gamma} \tilde{y}\left(D_{\Gamma(s)} \bar{\Phi}_{t}^{s}\right)^{-1}\left(D_{\Gamma(s)} \bar{\Phi}_{t}^{s}\right)^{-T} \nabla_{\Gamma} \psi \mathrm{d} \Gamma(s) \\
+\int_{\Gamma(s)} \tilde{y}\left(\phi_{t}^{s}\left(\operatorname{div}_{\Gamma(t)} V\right)+\tilde{b}\right) \psi \mathrm{d} \Gamma(s) \\
\quad-\int_{\Gamma(s)} \nabla_{\Gamma} \tilde{y}\left(D_{\Gamma(s)} \bar{\Phi}_{t}^{s}\right)^{-1}\left(D_{\Gamma(s)} \bar{\Phi}_{t}^{s}\right)^{-T} \nabla_{\Gamma} J_{t}^{s} \frac{\psi}{J_{t}^{s}} \mathrm{~d} \Gamma(s) .
\end{gathered}
$$

By Assumption 2.1 the bilinear form $\left(D_{\Gamma(s)} \bar{\Phi}_{t}^{s}\right)^{-1}[\gamma]\left(D_{\Gamma(s)} \bar{\Phi}_{t}^{s}\right)^{-T}[\gamma]$ is positive definite on the tangential space $T_{\gamma} \Gamma(s)$ uniformly in $s, t \in[0, T]$ and $\gamma \in \Gamma(s)$. Thus, there exists $c>0$ such that for some $k_{0} \geqslant 0$ one has $a(t, \psi, \psi)+k_{0}\|\psi\|_{L^{2}(\Gamma(s))} \geqslant c\|\psi\|_{H^{1}(\Gamma(s))}$. We are now in the situation to apply for example [16, Ch. III, Theorem 1.2], to obtain a unique solution $\tilde{y} \in W_{s}(0, T)$ to equation (3.9) for initial data $\phi_{0}^{s} y_{0} \in L^{2}(\Gamma(s))$. Moreover the solution map is continuous

$$
\|\tilde{y}\|_{W_{S}(0, T)} \leqslant C\left(\|\tilde{f}\|_{L^{2}\left((0, T), H^{-1}(\Gamma(s))\right)}+\left\|\phi_{0}^{s} y_{0}\right\|_{L^{2}(\Gamma(s))}\right)
$$

Note again that $\|\tilde{f}\|_{L^{2}\left((0, T), H^{-1}(\Gamma(s))\right)} \leqslant C\|f\|_{L_{H^{-1}(\Gamma)}^{2}}$, since the multiplication with $J_{t}^{s}$ is a globally bounded linear homeomorphism in $H^{1}(\Gamma(s))$, as stated in the proof os Lemma 3.9.

The transformation of (3.8) into (3.9) works both ways, hence the uniqueness of $y \in W_{\Gamma}$. The norms can be estimated as in Lemma 3.2 and Lemma 3.9 and the theorem follows.

With regard to order-optimal convergence estimates, sometimes a slightly higher regularity than $y \in W_{\Gamma}$ is required. Assuming $f \in L_{L^{2}(\Gamma)}^{2}$ and $y_{0} \in H^{1}\left(\Gamma_{0}\right)$, one can apply a Galerkin approximation argument, see [3, Theorems 4.4 and 4.5] for manifolds or [10] for open sets, to obtain

$$
\|\dot{y}\|_{L_{L^{2}(\Gamma)}^{2}}^{2}+\sup _{t \in[0, T]}\left\|\nabla_{\Gamma(t)} y\right\|_{L^{2}(\Gamma(t))}^{2}+\int_{[0, T]}\|y\|_{H^{2}(\Gamma(t))}^{2} \mathrm{~d} t \leqslant C\left(\|y\|_{H^{1}(\Gamma(0))}^{2}+\|f\|_{L_{L^{2}(\Gamma)}^{2}}^{2}\right) .
$$

Note that from [17, Ch. I, Theorem 3.1] it then follows that $\phi_{t}^{s} y \in C\left([0, T], H^{1}(\Gamma(s))\right)$. 


\section{Control constrained optimal control problems}

Using the results from the previous section, we can now formulate control-constrained optimal control problems known for stationary domains, see for example [16] or [24]. We consider here the case of a distributed control $u \in L_{L^{2}(\Gamma)}^{2}$. In comparison to the case of controls on euclidean open sets these controls are easier to implement in practice because all points on the surface are accessible from the outside, i.e., the surrounding euclidean space. As to the practical relevance of the (archetypical) problems under consideration observe that in addition to being of interest in their own right from an engineering point of view they also can be seen as Tikhonov-regularized parameter identification problems. Their unregularized, ill-posed counterparts then correspond to the limiting case where the Tikhonov parameter $\alpha \geqslant 0$ vanishes.

In our first example, given a moving surface as in Assumption 2.1, let $S_{T}: L_{L^{2}(\Gamma)}^{2} \rightarrow$ $L^{2}(\Gamma(T))$ denote the solution operator $u \mapsto y(T)$, where $y$ satisfies

$$
\frac{\mathrm{d}}{\mathrm{d} t} \int_{\Gamma(t)} y \varphi \mathrm{d} \Gamma(t)+\int_{\Gamma(t)} \nabla_{\Gamma} y \cdot \nabla_{\Gamma} \varphi \mathrm{d} \Gamma(t)=\langle\dot{\varphi}, y\rangle_{H^{-1}(\Gamma(t)), H^{1}(\Gamma(t))}+\langle u, \varphi\rangle_{L_{L^{2}(\Gamma)}^{2}},
$$

for all $\varphi \in W_{\Gamma}$, and with $y(0)=0 \in L^{2}\left(\Gamma_{0}\right)$. We know, that every function $y \in W_{\Gamma}$ has a representation in $C\left([0, T], L^{2}(\Gamma(s))\right)$ for any $s \in[0, T]$, compare Lemma 2.2, and the inclusion $\phi_{(\cdot)}^{s} W_{\Gamma} \subset C\left([0, T], L^{2}(\Gamma(s))\right)$ is continuous (in fact compact). Thus $S_{T}$ is a continuous linear operator. Consider the Control problem

$$
\left(\mathbb{P}_{T}\right)\left\{\begin{array}{l}
\min _{u \in L_{L^{2}(\Gamma)}^{2}} \mathcal{O}(u):=\frac{1}{2}\left\|S_{T}(u)-y_{T}\right\|_{L^{2}(\Gamma(T))}^{2}+\frac{\alpha}{2}\|u\|_{L_{L^{2}(\Gamma)}^{2}}^{2} \\
\text { s.t. } a \leqslant u \leqslant b,
\end{array}\right.
$$

with $\alpha, a, b \in \mathbb{R}, a<b, \alpha>0$, and $y_{T} \in L^{2}(\Gamma(T))$. This is now a well posed problem. By standard arguments, see for example [24, Theorem 3.15], using the weak lower semicontinuity of $\mathcal{O}(\cdot)$, one can conclude the existence of a unique solution $u \in L_{L^{2}(\Gamma)}^{2}$.

For an other example let the linear continuous solution operator $S_{d}: L_{L^{2}(\Gamma)}^{2} \rightarrow L_{L^{2}(\Gamma)}^{2}, u \mapsto$ $y$, where $y$ solves (4.1), and consider the problem

$$
\left(\mathbb{P}_{d}\right)\left\{\begin{array}{l}
\min _{u \in L_{L^{2}(\Gamma)}^{2}} \mathcal{O}(u):=\frac{1}{2}\left\|S_{d}(u)-y_{d}\right\|_{L_{L^{2}(\Gamma)}^{2}}^{2}+\frac{\alpha}{2}\|u\|_{L_{L^{2}(\Gamma)}^{2}}^{2} \\
\text { s.t. } a \leqslant u \leqslant b,
\end{array}\right.
$$

with $\alpha, a, b$ as above and $y_{d} \in L_{L^{2}(\Gamma)}^{2}$. Again there exists a unique solution, see [24, Theorem 3.16].

The first order necessary optimality condition for $\left(\mathbb{P}_{d}\right)$ reads

$$
\left\langle S_{d} u-y_{d}, S_{d}(v-u)\right\rangle_{L_{L^{2}(\Gamma)}^{2}}+\alpha\langle u, v-u\rangle_{L_{L^{2}(\Gamma)}^{2}}=\left\langle\alpha u+S_{d}^{*}\left(S_{d} u-y_{d}\right), v-u\right\rangle_{L_{L^{2}(\Gamma)}^{2}} \geqslant 0,
$$

for all $v \in U_{\mathrm{ad}}=\left\{v \in L_{L^{2}(\Gamma)}^{2} \mid a \leqslant v \leqslant b\right\}$. The adjoint operator $S_{d}^{*}: L_{L^{2}(\Gamma)}^{2} \rightarrow L_{L^{2}(\Gamma)}^{2}$ maps $v \in L_{L^{2}(\Gamma)}^{2}$ onto the solution $p \in W_{\Gamma}$ of

$$
-\langle\dot{p}, \varphi\rangle_{H^{-1}(\Gamma(t)), H^{1}(\Gamma(t))}+\int_{\Gamma(t)} \nabla_{\Gamma} p \cdot \nabla_{\Gamma} \varphi \mathrm{d} \Gamma(t)=\langle v, \varphi\rangle_{L_{L^{2}(\Gamma)}^{2}},
$$


for all $\varphi \in W_{\Gamma}$, and $p(T)=0 \in L^{2}(\Gamma(T))$. This follows if one tests (4.1) with $p$ and (4.3) with $y$. Integrate over $[0, T]$ and use $y(0)=0$ and $p(T)=0$ to arrive at $\langle v, y\rangle_{L_{L^{2}(\Gamma)}^{2}}=\langle p, u\rangle_{L_{L^{2}(\Gamma)}^{2}}$, for $u, v \in L_{L^{2}(\Gamma)}^{2}$ arbitrary.

Note that via the time transform $t^{\prime}=T-t$ Equation (4.3) converts into equation (3.8) with $b=-\operatorname{div} \Gamma(t) V$. Therefore all the results from Section 3 also apply to (4.3).

The necessary condition (4.2) characterizes the optimum $u$ as the orthogonal projection of $-\frac{1}{\alpha} S_{d}^{*}\left(S_{d} u-y_{d}\right)$ onto $U_{\mathrm{ad}}$. In our situation this is the pointwise application of the orthogonal projection $P_{[a, b]}: \mathbb{R} \rightarrow[a, b]$, as one easily shows by standard arguments.

Thus, introducing the adjoint state $p_{d}(u)=S_{d}^{*}\left(S_{d} u-y_{d}\right)$, we can rewrite (4.2) as

$$
u=P_{[a, b]}\left(-\frac{1}{\alpha} p_{d}(u)\right) .
$$

Similarly the unique solution $\mathrm{u}$ of $\left(\mathbb{P}_{T}\right)$ is characterized by $u=P_{[a, b]}\left(-\frac{1}{\alpha} p_{T}(u)\right)$, with $p_{T}(u)=$ $S_{T}^{*}\left(S_{T} u-y_{T}\right)$. Note that however the adjoint state $p_{T}$ in general is less smooth than $p_{d}$. This is because the adjoint equation, i.e. the equation describing $S_{T}^{*}: L^{2}(\Gamma(T)) \rightarrow L_{L^{2}(\Gamma)}^{2}, v \mapsto p$, reads

$$
-\langle\dot{p}, \varphi\rangle_{H^{-1}(\Gamma(t)), H^{1}(\Gamma(t))}+\int_{\Gamma(t)} \nabla_{\Gamma} p \cdot \nabla_{\Gamma} \varphi \mathrm{d} \Gamma(t)=0,
$$

for all $\varphi \in W_{\Gamma}$ and with $p(T)=v \in L^{2}(\Gamma(T))$. While Theorem 3.12 applies, this is not the case for the smoothness assertion (3.10), as long as $y_{d} \in L^{2}(\Gamma(T)) \backslash H^{1}(\Gamma(T))$.

Before we can discuss the discretized control problems in Section 7, in the next two sections we present some results on the discretization of the state equation.

\section{Finite element discretization}

We now discretize $\Gamma$ using an approximation $\Gamma_{0}^{h}$ of $\Gamma_{0}$ which is globally of class $C^{0,1}$. For the sake of convenience let us assume $n=2$, i.e. $\Gamma(t)$ is a hypersurface in $\mathbb{R}^{3}$.

Following [8] and [3], we consider $\Gamma_{0}^{h}=\bigcup_{i \in I_{h}} T_{h}^{i}$ consisting of triangles $T_{h}^{i}$ with corners on $\Gamma_{0}$, whose maximum diameter is denoted by $h$. With FEM error bounds in mind we assume the family of triangulations $\left\{\Gamma_{0}^{h}\right\}_{h>0}$ to be regular in the usual sense that the angles of all triangles are bounded away from zero uniformly in $h$.

As detailed in [4] and [3] an evolving triangulation $\Gamma^{h}(t)$ of $\Gamma(t)$ is obtained by subjecting the vertices of $\Gamma_{0}^{h}$ to the flow $\bar{\Phi}$. Hence, the nodes of $\Gamma^{h}(t)$ reside on $\Gamma(t)$ for all times $t \in[0, T]$, the triangles $T_{h}^{i}$ being deformed into triangles $T_{h}^{i}(t)$ by the movement of the vertices. Let $m_{h}$ denote the number of vertices $\left\{X_{j}^{0}\right\}_{j=1}^{m_{h}}$ in $\Gamma_{0}^{h}$. Now $X_{j}(t)$ solves

$$
\frac{\mathrm{d}}{\mathrm{d} t} X_{j}(t)=V\left(X_{j}(t), t\right), \quad X_{j}(0)=X_{j}^{0} .
$$

Consider the finite element space

$$
Y_{h}(t)=\left\{\varphi \in L^{2}\left(\Gamma^{h}(t)\right) \mid \varphi \in C\left(\Gamma^{h}(t)\right) \text { and } \forall i \in I_{h}:\left.\varphi\right|_{T_{h}^{i}(t)} \in \Pi^{1}\left(T_{h}^{i}(t)\right)\right\}
$$

of piecewise linear, globally continuous functions on $\Gamma^{h}(t)$, and its nodal basis functions $\left\{\varphi_{j}(t)\right\}_{j=1}^{m_{h}}$ that are one at exactly one vertex $X_{i}(t)$ of $\Gamma^{h}(t)$ and zero at all others. While on $\Gamma^{h}(t)$ the notion 
of the space $H^{1}$ is a little bit more involved than in the smooth case we can still provide $Y_{h}(t)$ with an appropriate norm, i.e., for $\varphi \in Y_{h}(t)$ let

$$
\|\varphi\|_{Y_{h}(t)}^{2}=\int_{\Gamma^{h}(t)} \nabla_{\Gamma^{h}} \varphi \nabla_{\Gamma^{h}} \varphi+\varphi^{2} \mathrm{~d} \Gamma^{h}(t) .
$$

For the finite element approach, it is crucial for the triangles $T_{h}^{i}(t)$ not to degenerate while $\Gamma^{h}(t)$ evolves, which leads us to the following assumption.

ASSUMPTION 5.1 The angles of the triangles $T_{h}^{i}(t)$ are bounded away from zero, uniformly w.r.t. $h, i$ and $t$. Also assume $a_{t}\left(\Gamma^{h}(t)\right)=\Gamma(t)$, with the restriction of $a_{t}$ to $\Gamma^{h}(t)$ being a homeomorphism between $\Gamma^{h}(t)$ and $\Gamma(t)$.

While Assumption 5.1 may appear a rather strong one, a remeshing strategy using conformal mappings, e.g., on topological torii was devised in [9] that yields meshes satisfying the assumption. In order to ensure optimal approximation properties of the discretization of the surface, we require $d$ to be twice Lipschitz-continuously differentiable.

Assumption $5.2 d \in C^{2,1}\left(n_{T}\right)$.

Let us summarize some basic properties of the family $\left\{\Gamma^{h}(t)\right\}_{t \in[0, T]}$.

Definition 5.3 Let $\Phi_{\cdot, h}^{s}: \Gamma^{h}(s) \times[0, T] \rightarrow \mathbb{R}^{3}$ denote the flow of $\Gamma^{h}$, i.e. the unique continuous map, such that $\Phi_{t, h}^{s}\left(T_{h}^{i}(s)\right)=T_{h}^{i}(t)$ and $\Phi_{t, h}^{s}$ is affine linear on each $T_{h}^{i}(s)$. The velocity field of the triangulated surface $V_{h}=\partial_{t} \Phi_{t, h}^{0}$ is the piecewise linear interpolant of $V$ on each triangle $T_{h}^{i}(t)$.

As in Lemma 3.2 we define the pull-back $\phi_{t, h}^{s}: L^{2}\left(\Gamma^{h}(t)\right) \rightarrow L^{2}\left(\Gamma^{h}(s)\right), \phi_{t, h}^{s} v=v \circ \Phi_{s, h}^{t}$. Finally let $v^{h}(t)$ denote the normals of $\Gamma^{h}(t)$, defined on each $T_{h}^{i}(t)$.

LEMma 5.4 There holds $\Phi_{t, h}^{r}=\Phi_{t, h}^{s} \circ \Phi_{s, h}^{r}$ and thus $\Phi_{s, h}^{t} \circ \Phi_{t, h}^{s}=\mathrm{id}_{\Gamma^{h}(s)}$.

The piecewise constant Jacobian determinant $J_{t, h}^{s}$ of $\Phi_{t, h}^{s}$ satisfies for all $s, t \in[0, T]$

$$
\frac{1}{C_{J}^{h}} \leqslant \min _{\gamma \in \Gamma(s)} J_{t, h}^{s}(\gamma) \leqslant \max _{\gamma \in \Gamma(s)} J_{t, h}^{s}(\gamma) \leqslant C_{J}^{h}
$$

for some constant $C_{J}^{h}>0$ that does not depend on $h>0$. Moreover $J_{t, h}^{s}$ and $\mathrm{D}_{\Gamma^{h}(s)} \Phi_{t, h}^{s}$ : $T \Gamma^{h}(s) \rightarrow T \Gamma^{h}(t) \subset \mathbb{R}^{3}$ are differentiable with respect to time in the interior of each $T_{h}^{i}(s)$.

The nodal basis functions have the transport property

$$
\dot{\varphi}_{i}=\phi_{0, h}^{t} \frac{\mathrm{d}}{\mathrm{d} t} \phi_{t, h}^{0} \varphi_{i} \equiv 0, \quad 1 \leqslant i \leqslant m_{h} .
$$

Proof. Consider a Triangle $T_{h}^{i}(s), s \in[0, T]$. W.1.o.g. let $X_{1}(s), X_{2}(s), X_{3}(s)$ denote its vertices. Then, using matrices $X^{i}(t)=\left(X_{2}(t)-X_{1}(t), X_{3}(t)-X_{1}(t)\right)$, we can write $\gamma \in T_{h}^{i}(s)$ in reduced barycentric coordinates as $\lambda_{\gamma}(s)=\left(X^{i}(s)^{T} X^{i}(s)\right)^{-1} X^{i}(s)^{T}\left(\gamma-X_{1}(s)\right)$. On $T_{h}^{i}(s)$ the transformation $\Phi_{t, h}^{s}$ is uniquely defined by $\lambda_{\Phi_{t, h}^{s} \gamma}(t)=\lambda_{\gamma}(s)$ and thus

$$
\Phi_{t, h}^{s}(\gamma)=X^{i}(t)\left(X^{i}(s)^{T} X^{i}(s)\right)^{-1} X^{i}(s)^{T}\left(\gamma-X_{1}(s)\right)+X_{1}(t) .
$$


In the relative interior of $T_{h}^{i}(s)$ the map $\Phi_{t, h}^{s}: T_{h}^{i}(s) \rightarrow T_{h}^{i}(t)$ is differentiable and its derivative $\mathrm{D}_{T_{h}^{i}(s)} \Phi_{t, h}^{s}: \mathbb{R}^{3} \supset T T_{h}^{i}(s) \rightarrow T T_{h}^{i}(t) \subset \mathbb{R}^{3}$ can be represented in terms of the standard basis of $\mathbb{R}^{3}$ by the matrix $\left.D_{s, t}^{i}=X^{i}(t) X^{i}(s)^{T} X^{i}(s)\right)^{-1} X^{i}(s)^{T}$.

Now one easily proves that the angle condition in Assumption 5.1 ensures the existence of $c>0$ such that $\lambda^{T} X^{i}(s)^{T} X^{i}(s) \lambda \geqslant c \min \left(\left\|X_{2}(s)-X_{1}(s)\right\|^{2},\left\|X_{3}(s)-X_{1}(s)\right\|^{2}\right)\|\lambda\|^{2}$ for all $\lambda \in \mathbb{R}^{2}$, $s \in[0, T]$. Hence, $\left\|\left(X^{i}(s)^{T} X^{i}(s)\right)^{-1}\right\|_{2} \leqslant\left(c \min \left(\left\|X_{2}(s)-X_{1}(s)\right\|^{2},\left\|X_{3}(s)-X_{1}(s)\right\|^{2}\right)\right)^{-1}$, and since $\left\|X^{i}(s)^{T}\right\|_{2}^{2},\left\|X^{i}(s)\right\|_{2}^{2} \leqslant 2 \max \left(\left\|X_{2}(s)-X_{1}(s)\right\|^{2},\left\|X_{3}(s)-X_{1}(s)\right\|^{2}\right)$ we get

$$
\frac{\left\|\mathrm{D}_{T_{h}^{i}(s)} \Phi_{t, h}^{s} \mathrm{~d} \gamma\right\|}{\|\mathrm{d} \gamma\|} \leqslant C \frac{\max \left(\left\|X_{2}(s)-X_{1}(s)\right\|^{2},\left\|X_{3}(s)-X_{1}(s)\right\|^{2}\right)}{\min \left(\left\|X_{2}(s)-X_{1}(s)\right\|^{2},\left\|X_{3}(s)-X_{1}(s)\right\|^{2}\right.}
$$

for all $\mathrm{d} \gamma \in T T_{h}^{i}(s)$. Using again Assumption 5.1 one concludes that the quotient of edge lengths is uniformly bounded.

Also, one easily verifies for $r, t \in[0, T]$

$$
\Phi_{t, h}^{r} \gamma=\left(\Phi_{t, h}^{s} \circ \Phi_{s, h}^{r}\right) \gamma \text { and } \Phi_{s, h}^{t} \Phi_{t, h}^{s}=\operatorname{id}_{\Gamma^{h}(s)} .
$$

We have $\left.J_{t, h}^{s}\right|_{T_{h}^{i}(s)}=\sqrt{\left.\operatorname{det}\left(\mathfrak{B}(s)^{T} D_{T_{h}^{i}(s)} \Phi_{t, h}^{s}\right)^{T} D_{T_{h}^{i}(s)} \Phi_{t, h}^{s} \mathfrak{B}(s)\right)}$ on the triangle $T_{h}^{i}(s)$, where the derivative is represented with respect to an orthonormal basis $\mathfrak{B}(s)$ of $T T_{h}^{i}(s)$. As per above considerations the spectral radius of $D_{T_{h}^{i}(s)} \Phi_{t, h}^{s}$ is uniformly bounded. Hence, there exists $C_{J}^{h}>0$ such that $J_{t, h}^{s} \leqslant C_{J}^{h}$. Because we can switch $s$ and $t$ and since by (5.4) we have $\left(\Phi_{t, h}^{s}\right)^{-1}=\Phi_{s, h}^{t}$ and thus $\frac{1}{J_{t, h}^{s}}=J_{s, h}^{t} \leqslant C_{J}^{h}$ we conclude

$$
\forall s, t \in[0, T]: \forall \gamma \in \Gamma_{s}^{h} \frac{1}{C_{J}^{h}} \leqslant J_{t, h}^{s}(\gamma) \leqslant C_{J}^{h}
$$

The trajectories $\Phi_{t, h}^{s} \gamma, \gamma \in \Gamma^{h}(s)$, the Jacobian determinants $J_{t, h}^{s}$, and the entries of $D_{s, t}^{i}$ are differentiable for t, because the trajectories $X_{j}(t), 1 \leqslant j \leqslant m_{h}$ are, compare (5.1). Hence also $\mathrm{D}_{\Gamma(s)} \Phi_{t, h}^{s}$ is differentiable as a map into $\mathbb{R}^{3}$. The velocity $V_{h}(\gamma, s)=\partial_{t} \Phi_{t, h}^{s} \gamma$ equals $V$ at the vertices and depends linearly on the coordinates $\lambda_{\gamma}$. As for the transport property (5.3), it is a consequence of the piecewise linear transformations of the piecewise linear Ansatz functions $\varphi_{i}$ which implies $\phi_{t, h}^{0} \varphi_{i}(t)=\varphi_{i}(0)$, compare [3, Prop. 5.4].

REMARK 5.5 Similarly one can prove the map $\Phi_{t, h}^{s}: \Gamma^{h}(s) \rightarrow \Gamma^{h}(t)$ to be bi-Lipschitz with respect to the respective metrics. The Lipschitz constant $L$ does not depend on $s, t \in[0, T]$.

In order to compare functions defined on $\Gamma^{h}(t)$ with functions on $\Gamma(t)$, for sufficiently small $h>0$ we use the projection $a_{t}$ from (2.1) to lift a function $y \in L^{2}\left(\Gamma^{h}(t)\right)$ to $\Gamma(t)$

$$
y^{l}\left(a_{t}(x)\right)=y(x), \quad \forall x \in \Gamma^{h}(t),
$$

and for $y \in L^{2}(\Gamma(t))$ we define the inverse lift

$$
y_{l}(x)=y\left(a_{t}(x)\right), \quad \forall x \in \Gamma^{h}(t) .
$$


For small mesh parameters $h$ the lift operation $(\cdot)_{l}: L^{2}(\Gamma(t)) \rightarrow L^{2}\left(\Gamma^{h}\right)$ defines a linear homeomorphism with inverse $(\cdot)^{l}$. Moreover, there exists $c_{\text {int }}>0$ such that

$$
\max \left(\left|\left\|(\cdot)_{l}\right\|_{\left.\mathcal{L}\left(L^{2}(\Gamma(t))\right), L^{2}\left(\Gamma^{h}(t)\right)\right)}^{2}-1\right|,\left|\left\|(\cdot)^{l}\right\|_{\mathcal{L}\left(L^{2}\left(\Gamma^{h}(t)\right), L^{2}(\Gamma(t))\right)}^{2}-1\right|\right) \leqslant c_{\text {int }} h^{2},
$$

as shows the following lemma.

LEMMA 5.6 The restriction of $a_{t}$ to $\Gamma^{h}(t)$ is a piecewise diffeomorphism. Denote by $\delta_{h}$ the Jacobian determinant of $\left.a_{t}\right|_{\Gamma^{h}(t)}: \Gamma^{h}(t) \rightarrow \Gamma(t)$, i.e. $\delta_{h}=|\operatorname{det}(M)|$ where $M \in \mathbb{R}^{2 \times 2}$ represents the Derivative $\mathrm{d} a_{t}(x): T_{x} \Gamma^{h}(t) \rightarrow T_{a(x)} \Gamma(t)$ with respect to arbitrary orthonormal bases of the respective tangential space. For small $h>0$ there holds

$$
\sup _{t \in[0, T] \Gamma(t)} \sup _{\Gamma(t)}\left|1-\delta_{h}\right| \leqslant C h^{2},
$$

In particular $\left.a_{t}\right|_{\Gamma^{h}(t)}$ is a diffeomorphism on each triangle $T_{h}^{i}(t)$. Now $\frac{1}{\delta_{h}}=\left|\operatorname{det}\left(M^{-1}\right)\right|$, so that by the change of variable formula

$$
\left|\int_{\Gamma^{h}(t)} v_{l} \mathrm{~d} \Gamma^{h}(t)-\int_{\Gamma(t)} v \mathrm{~d} \Gamma(t)\right|=\left|\int_{\Gamma(t)} v \frac{1}{\delta_{h}^{l}}-v \mathrm{~d} \Gamma(t)\right| \leqslant c_{\text {int }} h^{2}\|v\|_{L^{1}(\Gamma)} .
$$

Also there exists $C>0$ such that

1. $\sup _{t \in[0, T]}\left\|\dot{\delta}_{h}(t)\right\|_{L^{\infty}\left(\Gamma^{h}(t)\right)} \leqslant C h^{2}$, where the material derivative is to be understood in the sense of $\Phi_{t, h}^{0}$ and

2. $\sup _{t \in[0, T]}\left\|\mathbb{P}\left(I-\mathbb{R}_{h}^{l}\right) \mathcal{P}\right\|_{L^{\infty}(\Gamma(t))} \leqslant C h^{2}$, where $\mathbb{R}_{h}=\frac{1}{\delta_{h}^{l}}(I-d \mathcal{H}) \mathbb{P}^{h}(I-d \mathcal{H}), \mathcal{H}_{i j}=$ $\partial_{x_{i} x_{j}} d$, and $\mathbb{P}=\left\{\delta_{i j}-v_{i} v_{j}\right\}_{i, j=1}^{n+1}$ and $\mathbb{P}^{h}=\left\{\delta_{i j}-v_{i}^{h} v_{j}^{h}\right\}_{i, j=1}^{n+1}$ are the projections on the respective tangential space.

Proof. A proof of assertion 1. can be found in [4, Lemma 5.4], for a proof of the rest of the lemma see [3, Lemma 5.1].

The next Lemma concerns the continuity of the lift operations between $L_{L^{2}\left(\Gamma^{h}\right)}^{2}$ and $L_{L^{2}(\Gamma)}^{2}$.

LEMma 5.7 Using the pull-back $\phi_{t, h}^{s}$ we can define $L_{L^{2}\left(\Gamma^{h}\right)}^{2}$ as in Definition 3.4. For sufficiently small $h>0$ the lift operation $(\cdot)^{l}$ constitutes a continuous isomorphism between $L_{L^{2}(\Gamma)}^{2}$ and $L_{L^{2}\left(\Gamma^{h}\right)}^{2}$ with inverse $(\cdot)_{l}$. There holds

$$
\left|\left\langle f_{l}, g_{l}\right\rangle_{L_{L^{2}\left(\Gamma^{h}\right)}^{2}}-\langle f, g\rangle_{L_{L^{2}(\Gamma)}^{2}}\right| \leqslant c_{\text {int }} h^{2}\left|\langle f, g\rangle_{L_{L^{2}(\Gamma)}^{2}}\right| .
$$

Proof. In order to define $L_{L^{2}\left(\Gamma^{h}\right)}^{2}$ it suffices to consider the action of $\Phi_{t, h}^{s}$ on each triangle $T_{h}^{i}(s)$ thus defining $L_{L^{2}\left(T_{h}^{i}\right)}^{2}$. Definition 3.4 applies since the restrictions of the flow are smooth. Because the edges are of Lebesgue measure zero we have $L^{2}(\Gamma(t))=\bigoplus_{i \in I_{h}} L^{2}\left(T_{h}^{i}(t)\right)$ and thus canonically it follows $L_{L^{2}\left(\Gamma^{h}\right)}^{2}=\bigoplus_{i \in I_{h}} L_{L^{2}\left(T_{h}^{i}(t)\right)}^{2}$ as well as $\int_{0}^{T}\langle\cdot, \cdot\rangle_{L^{2}\left(\Gamma^{h}(t)\right)} \mathrm{d} t=$ $\sum_{i \in I_{h}} \int_{0}^{T}\langle\cdot, \cdot\rangle_{L^{2}\left(T_{h}^{i}(t)\right)} \mathrm{d} t$ 
Let $\Psi_{t}=\Phi_{0}^{t} \circ a_{t} \circ \Phi_{t, h}^{0}$ denote the mapping between $\Gamma_{0}^{h}$ and $\Gamma_{0}$ induced by the projection $a_{t}$. By Assumption 5.2 and by the construction of $\bar{\Phi}_{t}^{0}$ and $\Phi_{t, h}^{0}$ is follows that $\Psi_{t}: \Gamma_{0}^{h} \rightarrow \Gamma_{0}$ is a diffeomorphism on each triangle $T_{h}^{i}(0)$ and globally one-to-one and onto. Also $\Psi_{t}$ and its spatial derivatives are continuous w.r.t. time $t$.

We will show that $\bar{\Psi}: \Gamma_{0}^{h} \times[0, T] \rightarrow \Gamma_{0} \times[0, T],(\gamma, t) \mapsto\left(\Psi_{t}(\gamma), t\right)$ is a piecewise diffeomorphism whose Jacobian determinant is bounded away from zero. By Assumption 5.1 we already have that $\bar{\Psi}$ is globally one-to-one. Together this implies that the pull-back with $\bar{\Psi}$ constitutes an isomorphism between $L^{2}\left(\Gamma_{0} \times[0, T]\right)$ and $L^{2}\left(\Gamma_{0}^{h} \times[0, T]\right)$. This again means that

$$
\phi_{t, h}^{0} f_{l} \in L^{2}\left([0, T], L^{2}\left(\Gamma_{0}^{h}\right)\right) \Leftrightarrow \phi_{t}^{0} f \in L^{2}\left([0, T], L^{2}\left(\Gamma_{0}\right)\right) .
$$

As to $\bar{\Psi}$ being al local diffeomorphism, the sets $\bar{T}_{h}^{i}=\bigcup_{t \in[0, T]} T_{h}^{i}(t)$ are a partition of $\Gamma_{0}^{h} \times[0, T]$. In the interior of each $\bar{T}_{h}^{i}$ the map $\bar{\Psi}$ is a diffeomorphism. In fact, let $\gamma \in \operatorname{int}\left(T_{h}^{i}\right)$ for some $1 \leqslant i \leqslant m_{h}$. Compute

$$
\mathrm{D}_{\Gamma_{0}^{h} \times[0, T]} \bar{\Psi}(\gamma)=\left(\begin{array}{cc}
\mathrm{D}_{\Gamma_{0}^{h}} \Psi_{t}(\gamma) & \partial_{t} \Psi_{t}(\gamma) \\
0 & 1
\end{array}\right)
$$

We have $\mathrm{D}_{\Gamma_{0}^{h}} \Psi_{t}=\mathrm{D}_{\Gamma(t)} \Phi_{0}^{t} \mathrm{D}_{\Gamma^{h}(t)} a_{t} \mathrm{D}_{\Gamma_{0}^{h}} \Phi_{t, h}^{0}$. Its Jacobian determinant is the product of the determinants $J_{0}^{t}, \delta_{h}$, and $J_{t, h}^{0}$ that are each bounded away from zero, uniformly in $\gamma$ and $t$, compare (5.2), and the Lemmas 5.6 and 3.2. Hence the Jacobian determinant of $\bar{\Psi}$ is bounded away from zero.

As to continuity of $(\cdot)_{l}$, by Lemma 5.6 we have that

$$
\left|\left\langle f_{l}, g_{l}\right\rangle_{L_{L^{2}\left(\Gamma^{h}\right)}^{2}}-\langle f, g\rangle_{L_{L^{2}(\Gamma)}^{2}}\right|=\left|\int_{[0, T]} \int_{\Gamma(t)} f g\left(\frac{1}{\delta_{h}^{l}}-1\right) \mathrm{d} \Gamma(t) \mathrm{d} t\right| \leqslant c_{\mathrm{int}} h^{2}\left|\langle f, g\rangle_{L_{\Gamma}^{2}}\right| .
$$

Now, instead of dealing with Problem (3.8) directly, w.l.o.g. we consider the equation

$$
\frac{\mathrm{d}}{\mathrm{d} t} \int_{\Gamma(t)} y \varphi \mathrm{d} \Gamma(t)+\int_{\Gamma(t)} \nabla_{\Gamma} y \cdot \nabla_{\Gamma} \varphi+\mu y \varphi \mathrm{d} \Gamma(t)=\langle\dot{\varphi}, y\rangle_{L^{2}(\Gamma(t))}+\langle f, \varphi\rangle_{L^{2}(\Gamma(t))},
$$

with $\bar{\mu} \in \mathbb{R}$ large enough to ensure $\mu:=b+\bar{\mu} \geqslant 1$. Note that $y$ solves (5.6) iff $e^{\bar{\mu} t} y$ solves (3.8) with right-hand side $e^{\bar{\mu} t} f$.

In order to formulate the space-discretization of (5.6), consider the trial space

$$
H_{Y_{h}}^{1}=\left\{\sum_{i=1}^{m_{h}} \bar{y}_{i}(t) \varphi_{i}(t) \in L_{L^{2}\left(\Gamma^{h}\right)}^{2} \mid \bar{y}_{i} \in H^{1}([0, T])\right\} \simeq H^{1}([0, T])^{m_{h}} .
$$

The following definition of weak material derivatives for functions in $H_{Y_{h}}^{1}$ exploits the fact that $H_{Y_{h}}^{1}$ is isomorph to $H^{1}([0, T])^{m_{h}}$. It thus avoids the issue of extending the theory from Section 3 for the smooth surfaces $\Gamma(t)$ to our Lipschitz approximations $\Gamma^{h}(t)$. 
LEMmA 5.8 The weak material derivative of $v=\sum_{i=1}^{m_{h}} \bar{v}_{i}(t) \varphi_{i}(t) \in H_{Y_{h}}^{1}$ is $\dot{v}=\phi_{0, h}^{t}\left(\phi_{t, h}^{0} v\right)^{\prime}=$ $\sum_{i=1}^{m_{h}} \bar{v}_{i}^{\prime}(t) \varphi_{i}(t)$. Let further $w \in H_{Y_{h}}^{1}$, then $\langle v, w\rangle_{L^{2}\left(\Gamma^{h}(t)\right)}$ is absolutely continuous and

$$
\frac{\mathrm{d}}{\mathrm{d} t} \int_{\Gamma^{h}(t)} v w \mathrm{~d} \Gamma^{h}(t)=\int_{\Gamma^{h}(t)} \dot{v} w+v \dot{w}+v w \operatorname{div} \Gamma_{h} V_{h} \mathrm{~d} \Gamma^{h}(t) .
$$

Proof. Observe $\dot{v}=\phi_{0, h}^{t}\left(\phi_{t, h}^{0} v\right)^{\prime}=\phi_{0, h}^{t}\left(\sum_{i=1}^{m_{h}} \bar{v}_{i}(t) \varphi_{i}(0)\right)^{\prime}=\phi_{0, h}^{t}\left(\sum_{i=1}^{m_{h}} \bar{v}_{i}^{\prime}(t) \varphi_{i}(0)\right)$ because $\left(\phi_{t, h}^{0} \varphi(t)\right)^{\prime}(\gamma)=\frac{\mathrm{d}}{\mathrm{d} t} \varphi_{i}(0)(\gamma)=0$ for all $\gamma \in \Gamma_{0}^{h}$, as in (5.3).

Apply Lemma 3.1 on each triangle to see that $\left\langle\varphi_{i}(t), \varphi_{j}(t)\right\rangle_{L^{2}\left(\Gamma^{h}(t)\right)}$ is smooth and

$$
\frac{\mathrm{d}}{\mathrm{d} t}\left\langle\varphi_{i}(t), \varphi_{j}(t)\right\rangle_{L^{2}\left(\Gamma^{h}(t)\right)}=\int_{\Gamma^{h}(t)} \varphi_{i} \varphi_{j} \operatorname{div} \Gamma_{h} V_{h} \mathrm{~d} \Gamma^{h}(t) .
$$

Now

$$
\langle v, w\rangle_{L^{2}\left(\Gamma^{h}(t)\right)}=\sum_{i, j=1}^{m_{h}} \bar{v}_{i}(t) \bar{w}_{j}(t)\left\langle\varphi_{i}(t), \varphi_{j}(t)\right\rangle_{L^{2}\left(\Gamma^{h}(t)\right)}
$$

and the second assertion follows, since $\bar{v}_{i}, \bar{w}_{j} \in H^{1}([0, T]), 1 \leqslant i, j \leqslant m_{h}$.

We approximate (5.6) by the following semi-discrete Problem. Consider a piecewise smooth, globally Lipschitz approximation $\lambda$ of $\mu_{l}$, such that $\lambda \geqslant 1$. Find $y \in H_{Y_{h}}^{1}$ such that for all $\varphi \in H_{Y_{h}}^{1}$

$\frac{\mathrm{d}}{\mathrm{d} t} \int_{\Gamma^{h}(t)} y_{h} \varphi \mathrm{d} \Gamma^{h}(t)+\int_{\Gamma^{h}(t)} \nabla_{\Gamma^{h}} y_{h} \cdot \nabla_{\Gamma^{h}} \varphi+\lambda y_{h} \varphi \mathrm{d} \Gamma^{h}(t)=\langle\dot{\varphi}, y\rangle_{L^{2}\left(\Gamma^{h}(t)\right)}+\left\langle f_{h}, \varphi\right\rangle_{L^{2}\left(\Gamma^{h}(t)\right)}$,

and $y_{h}(0)=y_{0}^{h} \in Y_{h}(0)$. One possible choice would be $\lambda=\mu_{l}, f_{h}=f_{l}$ and $y_{0}^{h}=P_{0}^{h}\left(\left(y_{0}\right)_{l}\right)$ with $P_{0}^{h}$ the $L^{2}\left(\Gamma_{0}^{h}\right)$-orthogonal projection onto $Y_{h}(0)$.

First of all let us state that (5.7) admits a unique solution in $H_{Y_{h}}^{1}$. This is because for $y_{h}=$ $\sum_{i=1}^{m_{h}} \bar{y}_{i} \varphi_{i}$ we can rewrite (5.7) as a smooth linear ODE with non-smooth inhomogeneity for the coefficient vector $\bar{y}=\left\{y_{i}\right\}_{i=1}^{m_{h}} \in H^{1}([0, T])^{m_{h}}$

$$
\frac{\mathrm{d}}{\mathrm{d} t}\left(M(t) \bar{y}_{h}(t)\right)+\left(A_{\lambda}(t)\right) \bar{y}(t)=F(t), \quad y_{h}(0)=y_{0}^{h},
$$

with smooth mass and stiffness matrices

$$
M(t)=\left\{\left\langle\varphi_{i}, \varphi_{j}\right\rangle_{L^{2}\left(\Gamma^{h}(t)\right)}\right\}_{i, j=1}^{m_{h}} \quad \text { and } \quad A_{\lambda}(t)=\left\{\int_{\Gamma^{h}(t)} \nabla_{\Gamma^{h}} \varphi_{i} \nabla_{\Gamma^{h}} \varphi_{j}+\lambda \varphi_{i} \varphi_{j} \mathrm{~d} \Gamma^{h}(t)\right\}_{i, j=1}^{m_{h}},
$$

and right-hand side $F(t)=\left\{\left\langle f_{l}, \varphi_{i}\right\rangle_{L^{2}\left(\Gamma^{h}(t)\right)}\right\}_{i=1}^{m_{h}} \in L^{2}\left([0, T], \mathbb{R}^{m_{h}}\right)$, compare also [3]. Observe that we used the continuity of the coefficients $\bar{y}_{i} \in H^{1}([0, T])$ as well as $\dot{\varphi}_{i}=0$. Existence of a solution $\bar{y}_{h} \in H^{1}([0, T])^{m_{h}}$ of (5.8) can be argued by variation of constants or, more generally, one can apply an existence result by Carathéodory, compare [1, Theorems 1.1 and 1.3]. Uniqueness of $y_{h}$ is a consequence of the following lemma. 
Lemma 5.9 (Stability) Let $y_{0} \in L^{2}\left(\Gamma_{0}\right)$ and $f \in L_{L^{2}(\Gamma)}^{2}$, and let $y_{h}$ solve (5.7) with $y_{0}^{h} \in Y_{h}(0)$ and $f_{h}=f_{l}$. There exists $C>0$, such that for sufficiently small $h>0$ the solution satisfies

$$
\left\|y_{h}\right\|_{L^{2}\left(\Gamma^{h}(T)\right)}^{2}+\int_{0}^{T} \int_{\Gamma^{h}}\left(\nabla_{\Gamma^{h}} y_{h}\right)^{2}+\lambda y_{h}^{2} \mathrm{~d} \Gamma^{h}(t) \mathrm{d} t \leqslant C\left(\left\|y_{0}^{h}\right\|_{L^{2}\left(\Gamma_{0}^{h}\right)}^{2}+\|f\|_{L_{L^{2}(\Gamma)}^{2}}^{2}\right),
$$

as well as

$$
\left\|\dot{y}_{h}\right\|_{L_{L^{2}\left(\Gamma^{h}\right)}^{2}}^{2}+\operatorname{ess} \sup _{t \in[0, T]} \int_{\Gamma^{h}}\left(\nabla_{\Gamma^{h}} y_{h}\right)^{2}+\lambda y_{h}^{2} \mathrm{~d} \Gamma^{h}(t) \leqslant C\left(\left\|y_{0}^{h}\right\|_{Y_{h}(0)}^{2}+\|f\|_{L_{L^{2}(\Gamma)}^{2}}^{2}\right) .
$$

The idea of the proof is the same as in the non-discretized case, see [3, Lemma 6.1].

Obviously the material derivative depends on the evolution of the surface, i.e. different derivatives arise according to whether $\phi_{t}^{s}$ or $\phi_{t, h}^{s}$ is applied to pull back a function to a fixed domain. In order to compare $\dot{z}_{h}^{l}$ with $\left(\dot{z}_{h}\right)^{l}$ we need the following lemma.

Lemma 5.10 Let $y=\sum_{i=1}^{m_{h}} \bar{y}_{i} \varphi_{i} \in H_{Y_{h}}^{1}$. The lift $y^{l}$ lies in $W_{\Gamma}$ with $\dot{y}^{l} \in L_{L^{2}(\Gamma)}^{2}$, and for a.e. $t \in[0, T]$ there holds

$$
\left|\dot{y}^{l}-(\dot{y})^{l}\right| \leqslant C h^{2}\left\|\nabla_{\Gamma(t)} y^{l}\right\|_{\mathbb{R}^{n+1}},
$$

a.e. on $\Gamma(t)$.

Proof. We start by computing the material derivatives of $\bar{\varphi}_{i}(x, t): \eta_{T} \rightarrow \mathbb{R}, \bar{\varphi}_{i}(x, t)=$ $\varphi_{i}^{l}\left(a_{t}(x), t\right)$, i.e. the constant extension of the trial function $\varphi_{i}, 1 \leqslant i \leqslant m_{h}$, along the normal field of $\Gamma(t)$, compare the proof of [3, Theorem 6.2]. Observe that $\varphi_{i}^{l}$ is not smooth along the edges of patches $a_{t}\left(T_{h}^{j}(t)\right)$. However, $\varphi_{i}^{l}$ is smooth in the (relative) interior of all $a_{t}\left(T_{h}^{j}(t)\right)$.

Differentiate $\bar{\varphi}_{i}$ at some point $\gamma \in \operatorname{relint}\left(T_{h}^{j}(t)\right)$ inside the relative interior of the facet to obtain

$$
\begin{aligned}
\nabla \bar{\varphi}_{i}(\gamma, t) & =\nabla \bar{\varphi}_{i}\left(a_{t}(\gamma), t\right)\left(I d-\nabla d(\gamma, t) \nabla d(\gamma, t)^{T}-d(\gamma, t) \nabla^{2} d(\gamma, t)\right), \\
\partial_{t} \bar{\varphi}_{i}(\gamma, t) & =\partial_{t} \bar{\varphi}\left(a_{t}(\gamma), t\right)+\nabla \bar{\varphi}_{i}\left(a_{t}(\gamma), t\right)\left(-\partial_{t} d(\gamma, t) \nabla d(\gamma, t)-d(\gamma, t) \partial_{t} \nabla d(\gamma, t)\right) .
\end{aligned}
$$

By construction of $\bar{\varphi}_{i}$ we have $\nabla \bar{\varphi}_{i}\left(a_{t}(\gamma)\right) \nabla d(\gamma, t)=\nabla_{\Gamma} \varphi_{i}^{l}\left(a_{t}(\gamma)\right) \nabla v\left(a_{t}(\gamma), t\right)=0$ since $\bar{\varphi}_{i}$ is constant along orthogonal lines through $\Gamma$. Also, from $d\left(\Phi_{t}^{0}(\gamma), t\right) \equiv 0$ it follows $\partial_{t} d=-\nabla d V$. The (strong) material derivatives do not depend on the extension $\bar{\varphi}_{i}$, but only on the values on $\Gamma$ and $\Gamma^{h}$, respectively. One gets $\dot{\varphi}_{i}^{l}\left(a_{t}(\gamma), t\right)=\partial_{t} \bar{\varphi}_{i}\left(a_{t}(\gamma), t\right)+\nabla \bar{\varphi}_{i}\left(a_{t}(\gamma), t\right) V\left(a_{t}(\gamma), t\right)$ and $\dot{\varphi}_{i}(\gamma, t)=\partial_{t} \bar{\varphi}_{i}(\gamma, t)+\nabla \bar{\varphi}_{i}(\gamma, t) V_{h}(\gamma, t)$ which together with (5.9) leads us to

$$
\dot{\varphi}_{i}^{l}=\left(\dot{\varphi}_{i}\right)^{l}+\left(V-V_{h}+d\left(\left(\nabla^{2} d\right) V_{h}+\partial_{t} \nabla d\right)\right) \nabla_{\Gamma(t)} \varphi_{i}^{l},
$$

in the relative interior of the patches $a_{t}\left(T_{h}^{j}(t)\right), j \in I_{h}$.

In order to prove that the pull-back $\tilde{\varphi}:=\phi_{t}^{0} \varphi_{i}^{l}$ lies in $C^{1}\left([0, T], L^{2}\left(\Gamma_{0}\right)\right) \cap C\left([0, T], H^{1}\left(\Gamma_{0}\right)\right)$ for all $1 \leqslant i \leqslant m_{h}$ we proceed in four steps.

1. We show that $\tilde{\varphi}$ is globally Lipschitz on $\Gamma_{0} \times[0, T]$. Observe, that (5.9) implies that all derivatives of $\tilde{\varphi}$ exist and are bounded on the interior of patches $P_{h}^{i}(t)=\Phi_{0}^{t}\left(a_{t}\left(T_{h}^{i}(t)\right)\right)$. Since $\Psi_{t}=$ $\Phi_{0}^{t} \circ a_{t} \circ \Phi_{t, h}^{0}: \Gamma_{0}^{h} \times[0, T] \rightarrow \Gamma_{0}$ smoothly maps the edges of $\Gamma_{0}^{h}$ into $\Gamma_{0}$ the domains 
$\bigcup_{t \in[0, T]} P_{h}^{i}(t) \times\{t\} \subset \Gamma_{0} \times[0, T]$ have piecewise $C^{1}$-boundaries. Also, $\tilde{\varphi}$ is continuous and we are in the situation to apply Stoke's theorem to confirm $\tilde{\varphi} \in W^{1, \infty}\left(\Gamma_{0} \times[0, T]\right)$. By Morrey's lemma, for a formulation on manifolds see [19], we conclude $\tilde{\varphi} \in C^{0,1}\left(\Gamma_{0} \times[0, T]\right)$.

2. Now as to the time derivative, fix $\epsilon>0$ and $t \in(0, T)$. Let $L>0$ denote the global Lipschitz constant of $\tilde{\varphi}$ on $\Gamma_{0} \times[0, T]$ and choose $\eta>0$ sufficiently small such that $\sum_{i \in I_{h}}$ meas $\left(P_{h}^{i} \backslash P_{h, \eta}^{i}\right) \leqslant \epsilon^{2} / 8 L^{2}$ where $P_{h, \eta}^{i}=\left\{\gamma \in P_{h}^{i} \mid B_{\eta}(\gamma) \subset P_{h}^{i}\right\}$, the balls $B_{\eta}(\gamma)$ being taken with respect to the metric of $\Gamma_{0}$. Now, as stated above, the patches $P_{h}^{i}(t)=\Psi(t)\left(T_{h}^{i}\right)$ move continuously across $\Gamma_{0}$, and we can choose $K$ sufficiently small such that for all $i \in I_{h}$ and $k \in(-K, K)$ we have $P_{h, \eta}^{i}(t) \subset P_{h}^{i}(t+k)$. The derivative $\partial_{t} \tilde{\varphi}(\gamma, t)=\phi_{t}^{0} \dot{\varphi}_{i}^{l}$ which is defined a.e. on $\Gamma_{0} \times[0, T]$ then is continuous on the compact set $\mathcal{K}_{\eta}=\bigcup_{i \in I_{h}} \overline{P_{h, \eta}^{i}(t)} \times[t-K, t+K]$ and we have

$$
\begin{aligned}
\frac{1}{k^{2}} \int_{\Gamma_{0}}\left(\tilde{\varphi}(t+k)-\tilde{\varphi}(t)-\partial_{t} \tilde{\varphi}(t) k\right)^{2} \mathrm{~d} \Gamma_{0}= & \frac{1}{k^{2}} \sum_{i \in I_{h}}\left(\int_{P_{h, \eta}^{i}}\left(\tilde{\varphi}(t+k)-\tilde{\varphi}(t)-\partial_{t} \tilde{\varphi}(t) k\right)^{2} \mathrm{~d} \Gamma_{0}\right. \\
& \left.+\int_{P_{h}^{i} \backslash P_{h, \eta}^{i}}\left(\tilde{\varphi}(t+k)-\tilde{\varphi}(t)-\partial_{t} \tilde{\varphi}(t) k\right)^{2} \mathrm{~d} \Gamma_{0}\right) .
\end{aligned}
$$

Substituting $\tilde{\varphi}(\gamma, t+k)-\tilde{\varphi}(\gamma, t)=\partial_{t} \tilde{\varphi}(\gamma, t) k+\int_{0}^{1}\left(\partial_{t} \tilde{\varphi}(\gamma, t+\tau k)-\partial_{t} \tilde{\varphi}(\gamma, t)\right) k \mathrm{~d} \tau$ on $P_{h, \epsilon}^{i}$ like in the proof of Lemma 3.7 we choose $k$ small enough for

$$
\sup _{\tau \in[0,1]}\left\|\partial_{t} \tilde{\varphi}(t+\tau k)-\partial_{t} \tilde{\varphi}(t)\right\|_{\infty}^{2} \leqslant \frac{\epsilon^{2}}{2 \operatorname{meas}\left(\Gamma_{0}\right)},
$$

which is possible by uniform continuity of $\partial_{t} \tilde{\varphi}$ on $\mathcal{K}_{\eta}$. Estimating the second addend by $(2 L k)^{2} \sum_{i \in I_{h}} \operatorname{meas}\left(P_{h}^{i} \backslash P_{h, \eta}^{i}\right) \leqslant \epsilon^{2} / 2$ yields

$$
\limsup _{k \rightarrow 0} \frac{1}{k}\left\|\tilde{\varphi}(t+k)-\tilde{\varphi}(t)-\partial_{t} \tilde{\varphi}(t) k\right\|_{L^{2}\left(\Gamma_{0}\right)} \leqslant \epsilon .
$$

for every $\epsilon>0$. Hence $\tilde{\varphi}$ is differentiable into $L^{2}\left(\Gamma_{0}\right)$ with derivative $\partial_{t} \tilde{\varphi}$.

3. Thus in order to show $\tilde{\varphi} \in C^{1}\left([0, T], L^{2}\left(\Gamma_{0}\right)\right)$ it remains to prove that $\partial_{t} \tilde{\varphi}:[0, T] \rightarrow L^{2}\left(\Gamma_{0}\right)$ is continuous. By (5.9) $\partial_{t} \tilde{\varphi}$ is essentially bounded on $\Gamma_{0} \times[0, T]$. Let $M=\left\|\partial_{t} \tilde{\varphi}\right\|_{L^{\infty}\left(\Gamma_{0} \times[0, T]\right)}$. For $\epsilon>0$ choose $\eta>0$ sufficiently small such that $\sum_{i \in I_{h}} \operatorname{meas}\left(P_{h}^{i} \backslash P_{h, \eta}^{i}\right) \leqslant \epsilon^{2} / 8 M^{2}$. As above, choose $K>0$ and $\mathcal{K}_{\eta}$ accordingly. Now, choosing $k>0$ small enough such that (5.11) holds one arrives at

$$
\begin{aligned}
\left\|\partial_{t} \tilde{\varphi}(t+k)-\partial_{t} \tilde{\varphi}(t)\right\|_{L^{2}\left(\Gamma_{0}\right)}^{2}=\sum_{i \in I_{h}}\left(\int_{P_{h, \eta}^{i}}\left(\partial_{t} \tilde{\varphi}(t+k)-\partial_{t} \tilde{\varphi}(t)\right)^{2} \mathrm{~d} \Gamma_{0}\right. \\
\left.\quad+\int_{P_{h}^{i} \backslash P_{h, \eta}^{i}}\left(\partial_{t} \tilde{\varphi}(t+k)-\partial_{t} \tilde{\varphi}(t)\right)^{2} \mathrm{~d} \Gamma_{0}\right) \leqslant \epsilon^{2} .
\end{aligned}
$$


4. Continuity of $\tilde{\varphi}:[0, T] \rightarrow H^{1}\left(\Gamma_{0}\right)$ follows similarly. In fact, the spatial partial derivatives of $\tilde{\varphi}$ exhibit the same piecewise smooth structure as $\partial_{t} \tilde{\varphi}$.

Finally, $\tilde{\varphi}=\phi_{t}^{0} \varphi_{i}^{l} \in C^{1}\left([0, T], L^{2}\left(\Gamma_{0}\right)\right) \cap C\left([0, T], H^{1}\right)$ implies $\bar{y}_{i} \phi_{t}^{0} \varphi_{i}^{l} \in W_{0}(0, T)$, and we conclude $y^{l} \in W_{\Gamma}$ as well as $\dot{y}^{l} \in L_{L^{2}(\Gamma)}^{2}$. The estimate now is a consequence of (5.10).

Before we proceed to the main result of this section, we need to understand the approximation of elliptic equations on $\Gamma(t)$ by finite elements on $\Gamma^{h}(t)$.

LeMma 5.11 For $t \in[0, T]$ and $g \in L^{2}(\Gamma(t)), g_{h} \in L^{2}\left(\Gamma^{h}(t)\right)$ consider

$$
\int_{\Gamma(t)} \nabla_{\Gamma} Z^{g} \cdot \nabla_{\Gamma} \varphi+\mu Z^{g} \varphi \mathrm{d} \Gamma(t)=\langle g, \varphi\rangle_{L^{2}(\Gamma(t))}, \quad \forall \varphi \in H^{1}(\Gamma(t))
$$

and

$$
\int_{\Gamma^{h}(t)} \nabla_{\Gamma^{h}} Z_{h}^{g^{h}} \cdot \nabla_{\Gamma^{h}} \varphi+\mu_{l} Z_{h}^{g^{h}} \varphi \mathrm{d} \Gamma^{h}(t)=\left\langle g_{h}, \varphi\right\rangle_{L^{2}\left(\Gamma^{h}(t)\right)}, \quad \forall \varphi \in Y_{h}(t)
$$

with unique solutions $Z^{g} \in H^{1}(\Gamma(t))$ and $Z_{h}^{g^{h}} \in Y_{h}(t)$. The solution operators $S(t)$ : $L^{2}(\Gamma(t)) \rightarrow L^{2}(\Gamma(t)), g \mapsto Z^{g}$ and $S_{h}(t): L^{2}\left(\Gamma^{h}(t)\right) \rightarrow Y_{h} \subset L^{2}\left(\Gamma^{h}(t)\right), g_{h} \mapsto Z_{h}^{g_{h}}$ are self-adjoint. There exists $C$ independent of $t \in[0, T]$ such that

1. $\forall \varphi \in Y_{h}(t):\left|\left\|\varphi^{l}\right\|_{H^{1}(\Gamma(t))}^{2}-\|\varphi\|_{\left.Y_{h}(t)\right)}^{2}\right| \leqslant C h^{2}\left\|\varphi^{l}\right\|_{H^{1}(\Gamma(t))}^{2}<\infty$ as well as

2. $\left\|(\cdot)^{l} S_{h}(t)(\cdot)^{l^{*}}-S(t)\right\|_{\mathcal{L}\left(L^{2}(\Gamma(t)), L^{2}(\Gamma(t))\right)} \leqslant C h^{2}$ and

3. $\left\|(\cdot)^{l} S_{h}(t)(\cdot)^{l^{*}}-S(t)\right\|_{\mathcal{L}\left(L^{2}(\Gamma(t)), H^{1}(\Gamma(t))\right)} \leqslant C h$.

Proof. The operators being well-defined and self-adjoint follows by standard arguments. Assertion 1. follows from Lemma 5.6[2.], since $\varphi^{l}$ is continuous and piecewise smooth on $\Gamma(t)$ and thus lies in $H^{1}(\Gamma(t))$ with

$$
\int_{\Gamma^{h}(t)}\left\|\nabla_{\Gamma^{h}} \varphi\right\|^{2} \mathrm{~d} \Gamma^{h}(t)=\int_{\Gamma(t)}\left\|\nabla_{\Gamma} \varphi^{l}\right\|^{2} \mathrm{~d} \Gamma(t)+\int_{\Gamma(t)} \nabla_{\Gamma} \varphi^{l}\left(\mathbb{R}_{h}^{l}-\mathrm{Id}\right) \nabla_{\Gamma} \varphi^{l} \mathrm{~d} \Gamma(t),
$$

for details see, for example, [3, Lemma 5.2] and proof.

For a proof of 2. and 3. see [8, Theorem 8] and the discussion of $(\cdot)_{l}$ and $(\cdot)^{l^{*}}$ preceding Lemma 4 in aforementioned article. The fact that $C$ does not depend on $t$ is a consequence of Assumption 2.1 and 5.1.

THEOREM 5.12 Let Assumption 2.1, 5.1 and 5.2 hold and let $y \in W_{\Gamma}$ solve (5.6) for some $f \in$ $L_{L^{2}(\Gamma)}^{2}, y_{0} \in H^{1}\left(\Gamma_{0}\right)$, such that (3.10) holds. Let $y_{h}$ solve (5.7) with $\lambda=\mu_{l}$ and $f_{h}=f_{l}$ and some approximation $y_{0}^{h}$ of $\left(y_{0}\right)_{l}$. There exists $C>0$ independent of $y$ and $h$ such that

$$
\left\|y_{h}^{l}-y\right\|_{L_{L^{2}(\Gamma)}^{2}}^{2} \leqslant C\left(\left\|y_{h}(0)-y_{l}(0)\right\|_{L^{2}\left(\Gamma_{0}^{h}\right)}^{2}+h^{4}\left(\left\|y_{0}\right\|_{H^{1}\left(\Gamma_{0}\right)}^{2}+\left\|y_{0}^{h}\right\|_{Y_{h}(0)}^{2}+\|f\|_{L_{L^{2}(\Gamma)}^{2}}^{2}\right)\right) .
$$

Proof. Define $z=S(t)\left(y_{h}^{l}-y\right)$ and $z_{h}=S_{h}(t)\left(\delta_{h}\left(y_{h}-y_{l}\right)\right)$ with $S(t)$ and $S_{h}(t)$ as in Lemma 5.11. Now $\delta_{h}\left(y_{h}-y_{l}\right)=(\cdot)^{l^{*}}\left(y_{h}^{l}-y\right)$ and hence it follows from Lemma 5.11 [2.] that

$$
\left\|z_{h}^{l}-z\right\|_{L^{2}(\Gamma(t))}=\left\|\left((\cdot)^{l} S_{h}(\cdot)^{l^{*}}-S\right)\left(y_{h}^{l}-y\right)\right\|_{L^{2}(\Gamma(t))} \leqslant C h^{2}\left\|y_{h}^{l}-y\right\|_{L^{2}(\Gamma(t))},
$$


Observe now for $z_{h}=\sum_{i=1}^{m_{h}} \bar{z}_{i} \varphi_{i}$ using Lemma 5.10 we get

$$
Y=\left\{\left\langle y_{h}^{l}-y, \varphi_{i}^{l}\right\rangle_{L^{2}(\Gamma(t))}\right\}_{i=1}^{m_{h}} \in H^{1}([0, T])^{m_{h}} \text {, and thus } \bar{z}=\left(A_{\lambda}\right)^{-1} Y \in H^{1}([0, T])^{m_{h}} .
$$

Hence $\bar{z} \in H_{Y_{h}}^{1}$ and again by Lemma $5.10 z_{h}^{l} \in W_{\Gamma}$ as well as $\dot{z}_{h}^{l}(t) \in L^{2}(\Gamma(t))$.

We can now test (5.6) with $z_{h}^{l}$, using (5.12) in the process, to obtain

$$
\begin{aligned}
\frac{\mathrm{d}}{\mathrm{d} t}\left\langle y, z_{h}^{l}\right\rangle_{L^{2}(\Gamma(t))}+\left\langle y, y_{h}^{l}-y\right\rangle_{L^{2}(\Gamma(t))}=\left\langle\dot{z}_{h}^{l}, y\right\rangle_{L^{2}(\Gamma(t))} & +\left\langle f, z_{h}^{l}\right\rangle_{L^{2}(\Gamma(t))} \\
& +\left\langle-\Delta_{\Gamma} y+\mu y, z-z_{h}^{l}\right\rangle_{L^{2}(\Gamma(t))},
\end{aligned}
$$

and testing (5.7) with $z_{h}$ gives

$$
\frac{\mathrm{d}}{\mathrm{d} t}\left\langle y_{h}, z_{h}\right\rangle_{L^{2}\left(\Gamma^{h}(t)\right)}+\left\langle y_{h}^{l}, y_{h}^{l}-y\right\rangle_{L^{2}(\Gamma(t))}=\left\langle\dot{z}_{h}, y_{h}\right\rangle_{L^{2}\left(\Gamma^{h}(t)\right)}+\left\langle f_{l}, z_{h}\right\rangle_{L^{2}\left(\Gamma^{h}(t)\right)} .
$$

Now, since the strong material derivative $\dot{\delta}_{h}$ exists and is continuous on each triangle $T_{h}^{i}(t)$, the scalar products $\left\langle\varphi_{i}, \varphi_{j} \delta_{h}\right\rangle_{L^{2}\left(\Gamma^{h}(t)\right)}, 1 \leqslant i, j \leqslant m_{h}$, are differentiable with

$$
\frac{\mathrm{d}}{\mathrm{d} t}\left\langle\varphi_{i}, \varphi_{j} \delta_{h}\right\rangle_{L^{2}\left(\Gamma^{h}(t)\right)}=\int_{\Gamma^{h}(t)} \delta_{h} \varphi_{i} \varphi_{j} \operatorname{div} \Gamma^{h} V_{h}+\dot{\delta}_{h} \varphi_{i} \varphi_{j} \mathrm{~d} \Gamma^{h}(t)
$$

and we have

$$
\begin{aligned}
\frac{\mathrm{d}}{\mathrm{d} t}\left\langle y_{h}^{l}, z_{h}^{l}\right\rangle_{L^{2}(\Gamma(t))}= & \frac{\mathrm{d}}{\mathrm{d} t}\left\langle y_{h}, z_{h} \delta_{h}\right\rangle_{L^{2}\left(\Gamma^{h}(t)\right)} \\
= & \frac{\mathrm{d}}{\mathrm{d} t}\left\langle y_{h}, z_{h}\right\rangle_{L^{2}\left(\Gamma^{h}(t)\right)}+\left\langle y_{h}, \dot{z}_{h}\left(\delta_{h}-1\right)\right\rangle_{L^{2}\left(\Gamma^{h}(t)\right)}+\left\langle y_{h}, z_{h} \dot{\delta}_{h}\right\rangle_{L^{2}\left(\Gamma^{h}(t)\right)} \\
& \quad+\left\langle\dot{y}_{h}, z_{h}\left(\delta_{h}-1\right)\right\rangle_{L^{2}\left(\Gamma^{h}(t)\right)}+\left\langle y_{h}, z_{h} \operatorname{div}_{\Gamma^{h}} V_{h}\left(\delta_{h}-1\right)\right\rangle_{L^{2}\left(\Gamma^{h}(t)\right)} .
\end{aligned}
$$

Hence, we can rewrite (5.16) by means of the $L^{2}(\Gamma(t))$

$$
\frac{\mathrm{d}}{\mathrm{d} t}\left\langle y_{h}^{l}, z_{h}^{l}\right\rangle_{L^{2}(\Gamma(t))}+\left\langle y_{h}^{l}, y_{h}^{l}-y\right\rangle_{L^{2}(\Gamma(t))}=\left\langle\left(\dot{z}_{h}\right)^{l}, y_{h}^{l}\right\rangle_{L^{2}(\Gamma(t))}+\left\langle f, z_{h}^{l}\right\rangle_{L^{2}(\Gamma(t))}+R^{h},
$$

with

$$
\begin{aligned}
R^{h}=\left\langle y_{h}, z_{h} \dot{\delta}_{h}\right\rangle_{L^{2}\left(\Gamma^{h}(t)\right)}+\left\langle\dot{y}_{h}, z_{h}\left(\delta_{h}-1\right)\right\rangle_{L^{2}\left(\Gamma^{h}(t)\right)}+\left\langle y_{h}, z_{h} \operatorname{div}_{\Gamma^{h}} V_{h}\left(\delta_{h}-1\right)\right\rangle_{L^{2}\left(\Gamma^{h}(t)\right)} \\
+\left\langle f_{l}, z_{h}\left(1-\delta_{h}\right)\right\rangle_{L^{2}\left(\Gamma^{h}(t)\right)} .
\end{aligned}
$$

Subtracting (5.15) from (5.17) yields

$$
\begin{aligned}
\frac{\mathrm{d}}{\mathrm{d} t}\left\langle y_{h}^{l}-y, z_{h}^{l}\right\rangle_{L^{2}(\Gamma(t))}+\left\|y_{h}^{l}-y\right\|_{L^{2}(\Gamma(t))}^{2} & \\
=\left\langle\left(\dot{z}_{h}\right)^{l}-\dot{z}_{h}^{l}, y\right\rangle_{L^{2}(\Gamma(t))}+\left\langle\dot{z}_{h},\right. & \left.\left(y_{h}-y_{l}\right) \delta_{h}\right\rangle_{L^{2}\left(\Gamma^{h}(t)\right)} \\
& +R^{h}+\left\langle-\Delta_{\Gamma} y+\mu y, z_{h}^{l}-z\right\rangle_{L^{2}(\Gamma(t))} .
\end{aligned}
$$


From (5.13) we know $\left\langle\dot{z}_{h}, \delta_{h}\left(y_{h}-y_{l}\right)\right\rangle_{L^{2}\left(\Gamma^{h}(t)\right)}=\bar{z}_{h}^{\prime} A_{\lambda} \bar{z}_{h}=\frac{1}{2} \frac{\mathrm{d}}{\mathrm{d} t}\left(\bar{z}_{h} A_{\lambda} \bar{z}_{h}\right)-\frac{1}{2} \bar{z}_{h} A_{\lambda}^{\prime}(t) \bar{z}_{h}$, in the notation of (5.8). Now, using (5.14) and

$$
\left|R^{h}\right| \leqslant C h^{2}\left\|z_{h}\right\|_{L^{2}\left(\Gamma^{h}(t)\right)}\left(\left\|y_{h}\right\|_{L^{2}\left(\Gamma^{h}(t)\right)}+\left\|\dot{y}_{h}\right\|_{L^{2}\left(\Gamma^{h}(t)\right)}+\left\|f_{l}\right\|_{L^{2}\left(\Gamma^{h}(t)\right)}\right)
$$

we can estimate

$$
\begin{aligned}
& \frac{1}{2} \frac{\mathrm{d}}{\mathrm{d} t}\left(\bar{z}_{h} A_{\lambda}(t) \bar{z}_{h}\right)+\left\|y_{h}^{l}-y\right\|_{L^{2}(\Gamma(t))}^{2} \\
& \leqslant C\left(h^{2}\|y\|_{L^{2}(\Gamma(t))}\left\|\nabla_{\Gamma^{h}(t)^{h}}\right\|_{\left(L^{2}\left(\Gamma^{h}(t)\right)\right)^{n=1}}\right. \\
& \left.\quad+\left\|z_{h}\right\|_{Y_{h}(t)}^{2}+h^{2}\|y\|_{H^{2}(\Gamma(t))}\left\|y_{h}-y_{l}\right\|_{L^{2}\left(\Gamma^{h}(t)\right)}\right)+\left|R^{h}\right| \\
& \leqslant \frac{1}{2}\left\|y_{h}-y_{l}\right\|_{L^{2}\left(\Gamma^{h}(t)\right)}^{2}+C\left(\bar{z}_{h} A_{\lambda}(t) \bar{z}_{h}\right. \\
& \left.\quad+h^{4}\left(\left\|y_{h}\right\|_{L^{2}\left(\Gamma^{h}(t)\right)}^{2}+\left\|\dot{y}_{h}\right\|_{L^{2}\left(\Gamma^{h}(t)\right)}^{2}+\left\|f_{l}\right\|_{L^{2}\left(\Gamma^{h}(t)\right)}^{2}+\|y\|_{H^{2}(\Gamma(t))}^{2}\right)\right) .
\end{aligned}
$$

We can now apply Gronwall's lemma for

$$
\begin{aligned}
& {\left[\bar{z}_{h} A_{\lambda}(t) \bar{z}_{h}\right]_{0}^{T}+\int_{[0, T]}\left\|y_{h}^{l}-y\right\|_{L^{2}(\Gamma(t))}^{2} \mathrm{~d} t} \\
& \quad \leqslant C h^{4} \int_{[0, T]}\left\|y_{h}\right\|_{L^{2}\left(\Gamma^{h}(t)\right)}^{2}+\left\|\dot{y}_{h}\right\|_{L^{2}\left(\Gamma^{h}(t)\right)}^{2}+\left\|f_{l}\right\|_{L^{2}\left(\Gamma^{h}(t)\right)}^{2}+\|y\|_{H^{2}(\Gamma(t))}^{2} \mathrm{~d} t,
\end{aligned}
$$

and with the stability estimate (3.10) and the Lemmas 5.9 and 5.7 we finally arrive at

$$
\begin{aligned}
\int_{[0, T]}\left\|y_{h}^{l}-y\right\|_{L^{2}(\Gamma(t))}^{2} \mathrm{~d} t \leqslant & C \overbrace{\int_{\Gamma_{0}^{h}\left(\nabla_{\Gamma_{0}^{h}} z_{h}\right)^{2}+\lambda z_{h}^{2} \mathrm{~d} \Gamma_{0}^{h}}^{=\left\langle y_{h}^{l}(0)-y(0), z_{h}\right\rangle_{L^{2}\left(\Gamma_{0}\right)}}} \\
& \left.+h^{4}\left(\left\|y_{0}\right\|_{H^{1}\left(\Gamma_{0}\right)}^{2}+\left\|y_{0}^{h}\right\|_{Y_{h}(0)}^{2}+\|f\|_{L_{L^{2}(\Gamma)}^{2}}^{2}\right)\right) .
\end{aligned}
$$

Apply again (5.14) to prove the lemma.

REMARK 5.13 Depending on the regularity of $y_{0}$, possible choices of $y_{0}^{h}$ yielding $\mathcal{O}\left(h^{2}\right)$ convergence of $y_{h}^{l}$ comprehend the piecewise interpolation of $\left(y_{0}\right)_{l}$ and the $L^{2}\left(\Gamma_{0}\right)$-orthogonal projection of $\left(y_{0}\right)_{l}$ onto $Y_{h}(0)$. For the latter, the term involving $z_{h}$ in (5.19) vanishes completely, but it's $H^{1}\left(\Gamma_{0}\right)$-stability requires further investigation.

The order of convergence is lower, if the solution of (5.6) does not satisfy the additional regularity estimate (3.10).

Theorem 5.14 Let Assumption 2.1, 5.1 and 5.2 hold and let $y \in W_{\Gamma}$ solve (5.6) for $f \equiv 0$, and $y_{0} \in L^{2}\left(\Gamma_{0}\right)$. There exists $C>0$ independent of $y$ and $h$ such that for the solution $y_{h}$ of (5.7) with $y_{0}^{h}=P_{0}^{h}\left(\left(y_{0}\right)_{l}\right)$ and $f_{h} \equiv 0$ there holds

$$
\left\|y_{h}^{l}-y\right\|_{L_{L^{2}(\Gamma)}^{2}}^{2} \leqslant C\left(h^{2}+\sup _{t \in[0, T]}\left\|\lambda^{l}-\mu\right\|_{L^{\infty}(\Gamma(t))}^{2}\right)\left\|y_{0}\right\|_{L^{2}\left(\Gamma_{0}\right)}^{2} .
$$


Proof. We proceed as in the proof of Theorem 5.12 up to (5.15) which now reads

$$
\begin{aligned}
\frac{\mathrm{d}}{\mathrm{d} t}\left\langle y, z_{h}^{l}\right\rangle_{L^{2}(\Gamma(t))}+\left\langle y, y_{h}^{l}-y\right\rangle_{L^{2}(\Gamma(t))} & \\
& =\left\langle z_{h}^{l}, y\right\rangle_{L^{2}(\Gamma(t))}+\left\langle-\Delta_{\Gamma} y+\mu y, z-z_{h}^{l}\right\rangle_{H^{-1}(\Gamma(t)), H^{1}(\Gamma(t))},
\end{aligned}
$$

Analogously to (5.14) we can apply Lemma 5.11[3.] and estimate the last term through

$$
\begin{aligned}
\left|\left\langle-\Delta_{\Gamma} y+\lambda y, z_{h}^{l}-z\right\rangle_{H^{-1}(\Gamma(t)), H^{1}(\Gamma(t))}\right| & \leqslant\left\|-\Delta_{\Gamma} y+\lambda y\right\|_{H^{-1}(\Gamma(t))}\left\|z_{h}^{l}-z\right\|_{H^{1}(\Gamma(t))} \\
& \leqslant\left\|-\Delta_{\Gamma} y+\lambda y\right\|_{H^{-1}(\Gamma(t))} C h\left\|y_{h}^{l}-y\right\|_{L^{2}(\Gamma(t))} .
\end{aligned}
$$

On the other hand (5.17) becomes

$$
\frac{\mathrm{d}}{\mathrm{d} t}\left\langle y_{h}^{l}, z_{h}^{l}\right\rangle_{L^{2}(\Gamma(t))}+\left\langle y_{h}^{l}, y_{h}^{l}-y\right\rangle_{L^{2}(\Gamma(t))}=\left\langle\left(\dot{z}_{h}\right)^{l}, y_{h}^{l}\right\rangle_{L^{2}(\Gamma(t))}+\left\langle\left(\mu_{l}-\lambda\right) y_{h}, z_{h}\right\rangle_{L^{2}\left(\Gamma^{h}(t)\right)}+R^{h} .
$$

Continue as in the proof of Theorem 5.12 to finally arrive at the analogue of (5.18)

$$
\begin{aligned}
& {\left[\bar{z}_{h} A_{\lambda}(t) \bar{z}_{h}\right]_{0}^{T}+\int_{[0, T]}\left\|y_{h}^{l}-y\right\|_{L^{2}(\Gamma(t))}^{2} \mathrm{~d} t} \\
& \leqslant C\left(h^{4}+\sup _{t \in[0, T]}\left\|\lambda^{l}-\mu\right\|_{L^{\infty}(\Gamma(t))}^{2}\right) \int_{[0, T]}\left\|y_{h}\right\|_{L^{2}\left(\Gamma^{h}(t)\right)}^{2} \mathrm{~d} t \\
& +C h^{2} \int_{[0, T]}\|y\|_{H^{1}(\Gamma(t))}^{2}+h^{2}\left\|\dot{y}_{h}\right\|_{L^{2}\left(\Gamma^{h}(t)\right)}^{2} \mathrm{~d} t .
\end{aligned}
$$

Note that due to Lemma 5.6

$$
\left|\bar{z}_{h}(0) A_{\lambda}(0) \bar{z}_{h}(0)\right|=\left|\left\langle y_{h}^{l}(0)-y(0), z_{h}\right\rangle_{L^{2}\left(\Gamma_{0}\right)}\right| \leqslant \overbrace{\left\langle y_{h}(0)-y_{l}(0), z_{h}\right\rangle_{L^{2}\left(\Gamma_{0}^{h}\right)} \mid}^{=0 \text { since } y_{0}^{h}=P_{0}^{h}\left(\left(y_{0}\right)_{l}\right)}+C h^{2}\left\|y_{0}\right\|_{L^{2}\left(\Gamma_{0}\right)}^{2} .
$$

In view of Lemma 5.9 it remains to bound $\int_{0}^{T} h^{2}\left\|\dot{y}_{h}\right\|_{L^{2}\left(\Gamma^{h}(t)\right)}^{2} \mathrm{~d} t$. Again thanks to Lemma 5.9 we have

$$
\int_{0}^{T}\left\|\dot{y}_{h}\right\|_{L^{2}\left(\Gamma^{h}(t)\right)}^{2} \mathrm{~d} t \leqslant C\left\|y_{0}^{h}\right\|_{Y_{h}(0)}^{2} \text {. }
$$

But an inverse estimate, compare for example [2, Theorem 17.2], yields $\left\|y_{0}^{h}\right\|_{H^{1}\left(\Gamma_{0}^{h}\right)} \leqslant$ $\frac{C}{h}\left\|y_{0}^{h}\right\|_{L^{2}\left(\Gamma_{0}^{h}\right)}$, and because of the continuity of the lift $(\cdot)_{l}$ and of the $L^{2}$-projection $P_{0}^{h}$ the theorem follows.

\section{Implicit Euler discretization}

In order to solve (3.8) we apply a vertical method of lines. The time discretization is carried out by discontinuous Galerkin - implicit Euler discretization in $L_{L^{2}\left(\Gamma^{h}\right)}^{2}$. For $N \in \mathbb{N}$, consider an 
equidistant partition $I_{n}=\left(t_{n-1}, t_{n}\right]$ of $(0, T]$ with $1 \leqslant n \leqslant N, k=\frac{T}{N}$ and $t_{n}=k n$. The trial space for the discontinuous Galerkin method (DGM) is the space of 'piecewise constant' functions

$$
W_{k}^{h}=\left\{v \in L_{L^{2}\left(\Gamma^{h}\right)}^{2} \mid \forall 1 \leqslant n \leqslant N: \exists v^{n} \in Y_{h}\left(t_{n}\right): v \equiv \phi_{t_{n}, h}^{t} v^{n} \text { on } I_{n}\right\} .
$$

Note that in the following we will omit the operators $\phi_{t, h}^{s}$ when dealing with functions $w \in W_{k}^{h}$. Also, to further simplify notation let $\mathfrak{a}(t ; \psi, \varphi)=\int_{\Gamma^{h}(t)} \nabla_{\Gamma^{h}} \psi \cdot \nabla_{\Gamma^{h}} \varphi+\lambda \psi \varphi \mathrm{d} \Gamma^{h}(t)$ as well as $\langle\cdot, \cdot\rangle_{n}=\langle\cdot, \cdot\rangle_{L^{2}\left(\Gamma^{h}\left(t_{n}\right)\right)}$. W.1.o.g. we temporarily assume

$$
\inf _{t \in[0, T], \gamma \in \Gamma^{h}(t)} \lambda(\gamma, t)>M+2,
$$

with $M=\sup _{\tau \in[0, T]}\left\|\operatorname{div}_{\Gamma^{h}(\tau)} V_{h}\right\|_{L^{\infty}\left(\Gamma^{h}(\tau)\right)}$ such that

$$
\mathfrak{a}(t ; \varphi, \varphi)-M\|\varphi\|_{L^{2}\left(\Gamma^{h}(t)\right)}^{2} \geqslant\|\varphi\|_{Y_{h}(t)}^{2}+\|\varphi\|_{L^{2}\left(\Gamma^{h}(t)\right)}^{2}
$$

for all $t \in[0, T], h>0$ and all $\varphi \in Y_{h}(t)$.

To motivate the DGM insert the Ansatz $y_{h}^{k}(t)=\sum_{n=1}^{N} \phi_{t_{n}, h}^{t}\left(y^{n} \mathbf{1}_{I_{n}}\right) \in W_{k}^{h}$ with $y^{n} \in Y_{h}\left(t_{n}\right)$ into (5.7). If one understands the time-derivative in (5.7) in a distributional sense, the material derivative of $y_{h}^{k}$ becomes $\dot{y}_{h}^{k}=\sum_{n=1}^{N}\left(y^{n}-y^{n-1}\right) \delta_{t_{n-1}}$ and integration over time formally yields

$$
\left\langle y^{n}-y^{n-1}, \varphi\right\rangle_{n-1}+\int_{I_{n}} \mathfrak{a}\left(t ; y^{n}, \varphi\right)+\left\langle y^{n} \operatorname{div}_{\Gamma^{h}} V_{h}, \varphi\right\rangle_{L^{2}\left(\Gamma^{h}(t)\right)} \mathrm{d} t=\int_{I_{n}}\left\langle f_{h}, \varphi\right\rangle_{L^{2}\left(\Gamma^{h}(t)\right)} \mathrm{d} t,
$$

for smooth test functions $\varphi$. In order to arrive at a scheme that is symmetric with respect to test and ansatz space, we instead apply test functions $\varphi \in W_{k}^{h}$. At the discontinuities we set $\delta_{t_{n-1}} \varphi=\varphi^{n}$. Let it again be said that the above procedure is only a formal motivation for the shape of the method.

Using $\dot{y}^{n}=\dot{\varphi}^{n}=0$ one obtains

$$
\int_{I_{n}}\left\langle y^{n} \operatorname{div}_{\Gamma^{h}} V_{h}, \varphi\right\rangle_{L^{2}\left(\Gamma^{h}(t)\right)} \mathrm{d} t=\left\langle y^{n}, \varphi^{n}\right\rangle_{n}-\left\langle y^{n}, \varphi^{n}\right\rangle_{n-1} .
$$

Finally, to arrive at a computable scheme, lump the Integral over $\mathfrak{a}(t, \cdot, \cdot)$ and replace the right-hand side appropriately. For arbitrary parameters $y_{0}^{h} \in Y_{h}(0)$ and $f_{h} \in L_{L^{2}\left(\Gamma^{h}\right)}^{2}$ we rewrite the scheme as

$$
\begin{aligned}
y_{f}^{0}=y_{0}^{h}, \quad \forall \varphi \in W_{k}^{h}, \quad 1 \leqslant n \leqslant N: \\
\quad\left\langle y_{f}^{n}, \varphi^{n}\right\rangle_{n}-\left\langle y_{f}^{n-1}, \varphi^{n}\right\rangle_{n-1}+k \mathfrak{a}_{n}\left(y_{f}^{n}, \varphi^{n}\right)=\int_{I_{n}}\left\langle\phi_{t, h}^{t_{n}} f_{h}, \varphi^{n}\right\rangle_{n} \mathrm{~d} t,
\end{aligned}
$$

where $y_{0}^{h}, f_{h}$, and $\lambda$ are the same as in (5.7). For the approximation of the integral $\mathfrak{a}_{n}$ we assume $\mathfrak{a}_{n}(\psi, \varphi)=\mathfrak{a}\left(t_{n} ; \phi_{t, h}^{t_{n}} \psi, \phi_{t, h}^{t_{n}} \varphi\right)+\mathfrak{r}_{n}(\psi, \varphi)$, with a remainder

$$
\left|\mathfrak{r}_{n}(\psi, \varphi)\right| \leqslant C_{\mathfrak{r}} k\|\psi\|_{Y_{h}\left(t_{n}\right)}\|\varphi\|_{Y_{h}\left(t_{n}\right)} .
$$


One possible choice is $\mathfrak{r}_{n} \equiv 0$ for $1 \leqslant n \leqslant N$, but when it comes to approximating an adjoint equation such as (4.3) we will want to choose $\mathfrak{r}$ more freely. In order to proof convergence of the scheme (6.2) in $L_{L^{2}\left(\Gamma^{h}\right)}^{2}$ we make use of stability properties of the adjoint scheme

$$
\begin{aligned}
z_{g}^{N+1}=z_{T}, \quad & \forall \varphi \in W_{k}^{h}, \quad 1 \leqslant n \leqslant N: \\
& \left\langle z_{g}^{n}, \varphi^{n}\right\rangle_{n}-\left\langle z_{g}^{n+1}, \varphi^{n}\right\rangle_{n}+k \mathfrak{a}_{n}\left(\varphi^{n}, z_{g}^{n}\right)=\int_{I_{n}}\left\langle\phi_{t, h}^{t_{n}} g_{h}, \varphi^{n}\right\rangle_{n} \mathrm{~d} t .
\end{aligned}
$$

with $g_{h} \in L_{L^{2}\left(\Gamma^{h}\right)}^{2}, z_{T} \in Y_{h}(T)$. In Section 7 it will be important that given snapshots $\left\{\Gamma^{h}\left(t_{n}\right)\right\}_{n=1}^{N}$ of the surface (6.2) and (6.4) can be evaluated exactly for certain right-hand sides $f_{h}$ and $g_{h}$, e.g. $g_{h} \in W_{k}^{h}$. Let us introduce the mean value of a function $y \in L_{L^{2}\left(\Gamma^{h}\right)}^{2}$ over an interval $I_{n}$.

DEFinition 6.1 Let $\phi_{t, h}^{s}$ denote the pullback operator associated to the flow $\Phi_{t, h}^{s}$ as in Lemma 3.2 and let $s \in[0, T]$. The mean value of a function $y \in L_{L^{2}\left(\Gamma^{h}\right)}^{2}$ is defined as $\bar{y}^{n}(s)=\frac{1}{k} \int_{I_{n}} \phi_{t, h}^{s} y \mathrm{~d} t$ for $t \in I_{n}$. Because

$$
\int_{I_{n}} \phi_{t, h}^{s} y \mathrm{~d} t=\int_{I_{n}} \phi_{r, h}^{s} \phi_{t, h}^{r} y \mathrm{~d} t=\phi_{r, h}^{s} \underbrace{\int_{I_{n}} \phi_{t, h}^{r} y \mathrm{~d} t}_{\bar{y}_{n}(r)},
$$

$\bar{y}^{n}$ does not depend on $s \in[0, T]$.

Similarly one could define the mean value of $y \in W_{\Gamma}$ if one were to investigate a horizontal method-of-lines approach.

Now for $y_{0} \equiv 0, z_{T} \equiv 0$ the schemes are adjoint in the sense

$$
k \sum_{n=1}^{N}\left\langle\bar{f}_{h}^{n}, z_{g}\right\rangle_{n}=k \sum_{n=1}^{N}\left\langle\bar{g}_{h}^{n}, y_{f}\right\rangle_{n},
$$

i.e. the discrete solution operators $f_{h} \mapsto y_{f}$ and $g_{h} \mapsto z_{g}$ are adjoint as operators from $\left(L_{L^{2}\left(\Gamma^{h}\right)}^{2},\langle\cdot, \cdot\rangle_{h, k}\right)$ into itself, where $L_{L^{2}\left(\Gamma^{h}\right)}^{2}$ is equipped with the scalar product

$$
\langle f, g\rangle_{h, k}=k \sum_{n=1}^{N} \int_{I_{n}}\left\langle\left(\phi_{t, h}^{t_{n}} f\right),\left(\phi_{t, h}^{t_{n}} g\right)\right\rangle_{n} \mathrm{~d} t .
$$

LEMMA 6.2 Let $\|\cdot\|_{h, k}$ denote the norm induced by $\langle\cdot, \cdot\rangle_{h, k}$. The norms $\|\cdot\|_{L_{L^{2}\left(\Gamma^{h}\right)}^{2}}$ and $\|\cdot\|_{h, k}$ on $L_{L^{2}\left(\Gamma^{h}\right)}^{2}$ are equivalent and there holds

$$
\left|\langle f, g\rangle_{h, k}-\langle f, g\rangle_{L_{L^{2}\left(\Gamma^{h}\right)}^{2}}\right| \leqslant C k\left|\langle f, g\rangle_{L_{L^{2}\left(\Gamma^{h}\right)}^{2}}\right| .
$$

Proof. The result follows from the identity

$$
\int_{[0, T]} \int_{\Gamma^{h}(t)} f g \mathrm{~d} \Gamma^{h}(t) \mathrm{d} t=\sum_{n=1}^{N} \int_{I_{n}} \int_{\Gamma^{h}\left(t_{n}\right)}\left(\phi_{t, h}^{t_{n}} f\right)\left(\phi_{t, h}^{t_{n}} g\right) J_{t_{n}, h}^{t} \mathrm{~d} \Gamma^{h}\left(t_{n}\right) \mathrm{d} t,
$$

and $J_{t}^{t_{n}}$ being Lipschitz with $J_{t_{n}, h}^{t_{n}} \equiv 1$. 
Note also that for $z \in W_{k}^{h}$, since $\dot{z}^{n}=0$ on $I_{n}$, we can apply the mean value theorem to obtain for some $t \in I_{n}$

$$
\left|\left\|z^{n}\right\|_{L^{2}\left(\Gamma^{h}(t)\right)}^{2}-\left\|z^{n}\right\|_{n}^{2}\right|=k\left|\left\langle z^{n} \operatorname{div}_{\Gamma^{h}\left(\Theta_{n}\right)} V_{h}, z^{n}\right\rangle_{L^{2}\left(\Gamma^{h}\left(\Theta_{n}\right)\right)}\right| \leqslant M k\left\|z^{n}\right\|_{L^{2}\left(\Gamma^{h}\left(\Theta_{n}\right)\right)}^{2}
$$

with $\Theta_{n} \in\left(t, t_{n}\right)$. Apply (6.6) to itself to obtain for some $\tilde{\Theta}_{n} \in\left(\Theta_{n}, t_{n}\right)$

$$
\begin{aligned}
\left|\left\|z^{n}\right\|_{L^{2}\left(\Gamma^{h}(t)\right)}^{2}-\left\|z^{n}\right\|_{n}^{2}\right| & \leqslant M k\left(\left\|z^{n}\right\|_{n}^{2}+\left(\left\|z^{n}\right\|_{L^{2}\left(\Gamma^{h}\left(\Theta_{n}\right)\right)}^{2}-\left\|z^{n}\right\|_{n}^{2}\right)\right) \\
& \leqslant M k\left(\left\|z^{n}\right\|_{n}^{2}+M k\left\|z^{n}\right\|_{L^{2}\left(\Gamma^{h}\left(\tilde{\Theta}_{n}\right)\right)}^{2}\right) \\
& \leqslant M k\left(1+C_{L^{2}\left(\Gamma^{h}\right)} M k\right)\left\|z^{n}\right\|_{n}^{2} .
\end{aligned}
$$

A similar continuity assertion holds for the $Y_{h}(t)$-norm, as shows the following lemma.

Lemma 6.3 Let $y, z \in H_{Y_{h}}^{1}, \tilde{\lambda} \in C\left(\Gamma^{h}(s) \times[0, T]\right)$, and $\lambda=\phi_{s, h}^{t} \tilde{\lambda}$. There exists $C>0$ such that for every $s \in I_{n}$

$$
\left|\int_{I_{n}} \mathfrak{a}\left(s ; \phi_{t, h}^{s} y, \phi_{t, h}^{s} z\right) \mathrm{d} t-\int_{I_{n}} \mathfrak{a}(t ; y, z) \mathrm{d} t\right| \leqslant C k \int_{I_{n}}\left\|\phi_{t, h}^{s} y\right\|_{Y_{h}(s)}\left\|\phi_{t, h}^{s} z\right\|_{Y_{h}(s)} \mathrm{d} t
$$

i.e. for $z \in W_{k}^{h}$ we have

$$
\left|k \mathfrak{a}\left(s ; \bar{y}^{n}, z^{n}\right)-\int_{I_{n}} \mathfrak{a}(t ; y, z) \mathrm{d} t\right| \leqslant C k \int_{I_{n}}\left\|\phi_{t, h}^{s} y\right\|_{Y_{h}(s)}\left\|z^{n}\right\|_{Y_{h}(s)} \mathrm{d} t .
$$

In particular with $\lambda \equiv 1$ the estimates hold for $\mathfrak{a}(t ; \varphi, \varphi)=\|\varphi\|_{Y_{h}(t)}^{2}$.

Proof. We abbreviate $\tilde{\Delta}(s, t)=D_{\Gamma^{h}(s)} \Phi_{t, h}^{s}\left(D_{\Gamma^{h}(s)} \Phi_{t, h}^{s}\right)^{T} J_{t, h}^{s}$. Since $\dot{z}^{n} \equiv 0$ we have

$$
\begin{aligned}
& \left|\int_{I_{n}} \mathfrak{a}\left(s ; \phi_{t, h}^{s} y, \phi_{t, h}^{s} z\right) \mathrm{d} t-\int_{I_{n}} \mathfrak{a}(t ; y, z) \mathrm{d} t\right| \\
& =\left|\int_{I_{n}} \int_{\Gamma^{h}(s)} \nabla_{\Gamma^{h}} \phi_{t, h}^{s} y(\tilde{\Delta}(s, s)-\tilde{\Delta}(s, t)) \nabla_{\Gamma^{h}} \phi_{t, h}^{s} z+\lambda \phi_{t, h}^{s} y\left(J_{s, h}^{s}-J_{t, h}^{s}\right) \phi_{t, h}^{s} z \mathrm{~d} \Gamma^{h}(s) \mathrm{d} t\right|
\end{aligned}
$$

The lemma follows from the fact that $\Phi_{t, h}^{s}$ it linear on each $T_{h}^{i}(s)$ and globally Lipschitz in time, as by Lemma 5.4.

Let us formulate a crucial stability assertion for the adjoint scheme (6.4).

LEMmA 6.4 Let $z \in W_{k}^{h}$ solve (6.4) with right-hand side $g \in L_{L^{2}\left(\Gamma^{h}\right)}^{2}$ and final state $z_{T}=0$. For sufficiently small $k>0$ there exists $C>0$, depending only on $\Gamma$, such that

$$
\max _{1 \leqslant n \leqslant N} \mathfrak{a}\left(t_{n} ; z^{n}, z^{n}\right)+\frac{1}{k} \sum_{n=1}^{N}\left\|z^{n+1}-z^{n}\right\|_{n}^{2}+k \sum_{n=1}^{N}\left\|z^{n}\right\|_{H^{1}\left(\Gamma\left(t_{n}\right)\right)}^{2} \leqslant C\|g\|_{h, k}^{2} .
$$


Proof. Apply (6.4) to $z^{n}$ to obtain

$$
\left\langle z^{n}-z^{n+1}, z^{n}\right\rangle_{n}+k \mathfrak{a}_{n}\left(z^{n}, z^{n}\right)=\int_{I_{n}}\left\langle\phi_{t, h}^{t_{n}} g, z^{n}\right\rangle_{n} \mathrm{~d} t
$$

This leads to

$$
\begin{aligned}
& \frac{1}{2}\left(\left\|z^{n}\right\|_{n}^{2}+\left\|z^{n+1}-z^{n}\right\|_{n}^{2}-\left\|z^{n+1}\right\|_{n}^{2}\right)+k \mathfrak{a}_{n}\left(z^{n}, z^{n}\right)=\int_{I_{n}}\left\langle\phi_{t, h}^{t_{n}} g, z^{n}\right\rangle_{n} \mathrm{~d} t \\
& \leqslant \int_{I_{n}}\left\|\phi_{t, h}^{t_{n}} g\right\|_{L^{2}\left(\Gamma^{h}\left(t_{n}\right)\right)} \mathrm{d} t\left\|z^{n}\right\|_{n} \leqslant \frac{1}{2 k M}\left(\int_{I_{n}}\left\|\phi_{t, h}^{t_{n}} g\right\|_{n} \mathrm{~d} t\right)^{2}+\frac{k M}{2}\left\|z^{n}\right\|_{n}^{2} .
\end{aligned}
$$

Summing up and using (6.7) gives us

$$
\sum_{n=1}^{N}\left(\frac{1}{2}\left\|z^{n+1}-z^{n}\right\|_{n}^{2}-M k\left(1+\frac{1}{2} C_{L^{2}\left(\Gamma^{h}\right)} M k\right)\left\|z^{n}\right\|_{n}^{2}+k \mathfrak{a}_{n}\left(z^{n}, z^{n}\right)\right) \leqslant \frac{1}{2 M}\|g\|_{h, k}^{2},
$$

such that for $0<k<\min \left(\frac{2}{C_{L^{2}\left(\Gamma^{h}\right)^{M^{2}}}}, \frac{1}{2 C_{\mathrm{r}}}\right)$

$$
\begin{aligned}
\frac{k}{2} \sum_{n=1}^{N}\left\|z^{n}\right\|_{H^{1}\left(\Gamma\left(t_{n}\right)\right)}^{2} & \leqslant k \sum_{n=1}^{N}\left(\mathfrak{a}\left(t_{n} ; z^{n}, z^{n}\right)+\mathfrak{r}_{n}\left(z^{n}, z^{n}\right)-\left(1+\frac{C_{L^{2}\left(\Gamma^{h}\right)} M k}{2}\right) M\left\|z^{n}\right\|_{n}^{2}\right) \\
& \leqslant \frac{1}{2 M}\|g\|_{h, k}^{2} .
\end{aligned}
$$

Now we test (6.4) with $z^{n}-z^{n+1}$ to get

$$
\begin{aligned}
\left\|z^{n}-z^{n+1}\right\|_{n}^{2}+\frac{k}{2} & \left(\mathfrak{a}_{n}\left(z^{n}, z^{n}\right)+\mathfrak{a}_{n}\left(z^{n+1}-z^{n}, z^{n+1}-z^{n}\right)-\mathfrak{a}_{n}\left(z^{n+1}, z^{n+1}\right)\right) \\
& =\int_{I_{n}}\left\langle\phi_{t, h}^{t_{n}} g, z^{n}-z^{n+1}\right\rangle_{n} \mathrm{~d} t \leqslant \frac{1}{2}\left(\int_{I_{n}}\left\|\phi_{t, h}^{t_{n}} g\right\|_{n}^{2} \mathrm{~d} t\right)^{2}+\frac{1}{2}\left\|z^{n}-z^{n+1}\right\|_{n}^{2} .
\end{aligned}
$$

Summing up and using Lemma 6.3 on $\mathfrak{a}$ as well as the estimate (6.3) on $\mathfrak{r}$ we arrive at

$$
\begin{aligned}
& \frac{k}{2} \mathfrak{a}\left(t_{m}, z^{m}, z^{m}\right)+\frac{1}{2} \sum_{n=m}^{N}\left(\left\|z^{n+1}-z^{n}\right\|_{n}^{2}\right) \\
& \quad \leqslant \frac{1}{2} k\|g\|_{h, k}^{2}+\frac{k}{2} \sum_{n=m+1}^{N} \mathfrak{a}\left(t_{n-1} ; z^{n}, z^{n}\right)-\mathfrak{a}\left(t_{n} ; z^{n}, z^{n}\right)+\mathfrak{r}_{n-1}\left(z^{n}, z^{n}\right)-\mathfrak{r}_{n}\left(z^{n}, z^{n}\right) \\
& \quad \leqslant \frac{1}{2} k\|g\|_{h, k}^{2}+\frac{k}{2} \sum_{n=m+1}^{N} C k\left(\left\|z^{n}\right\|_{H^{1}\left(\Gamma\left(t_{n}\right)\right)}^{2}+\left\|z^{n}\right\|_{H^{1}\left(\Gamma\left(t_{n-1}\right)\right)}^{2}\right)
\end{aligned}
$$

Combine with (6.8) to arrive at the lemma. 
The following Lemma shows, that it is sufficient to estimate the approximation error at the points $t_{n}, 1 \leqslant n \leqslant N$ to prove convergence in $L_{L^{2}\left(\Gamma^{h}\right)}^{2}$.

LEMMA 6.5 Let $r \in H^{1}([0, T], V), V$ a separable Hilbert space, then there holds for $\tau \in I_{n}$

$$
\|r-r(\tau)\|_{L^{2}\left(I_{n}, V\right)} \leqslant k\left\|r^{\prime}\right\|_{L^{2}\left(I_{n}, V\right)} .
$$

In our situation this implies for $r \in H_{Y_{h}}^{1}$ that

1. $\left\|r(\tau)-\bar{r}^{n}\right\|_{L^{2}\left(\Gamma^{h}(\tau)\right)}^{2} \leqslant C k \int_{I_{n}}\|\dot{r}\|_{L^{2}\left(\Gamma^{h}(t)\right)}^{2} \mathrm{~d} t$,

2. and $\int_{I_{n}}\left\|r(t)-\bar{r}^{n}\right\|_{L^{2}\left(\Gamma^{h}(t)\right)}^{2} \mathrm{~d} t \leqslant C k^{2} \int_{I_{n}}\|\dot{r}\|_{L^{2}\left(\Gamma^{h}(t)\right)}^{2} \mathrm{~d} t$.

Proof. For the fist assertion approximate $r$ by $r_{i} \in \Phi([0, T], V)$ such that $r_{i} \stackrel{H^{1}([0, T], V)}{\longrightarrow} r$ as $i \rightarrow \infty$. Use

$$
\begin{aligned}
\left\|r_{i}-r_{i}(\tau)\right\|_{L^{2}\left(I_{n}, V\right)}=\left(\int_{I_{n}}\left\|\int_{\tau}^{t} r_{i}^{\prime}(\theta) \mathrm{d} \theta\right\|_{V}^{2} \mathrm{~d} t\right)^{\frac{1}{2}} & \\
& \leqslant\left(\int_{I_{n}} k \int_{I_{n}}\left\|r_{i}^{\prime}(\theta)\right\|_{V}^{2} \mathrm{~d} \theta \mathrm{d} t\right)^{\frac{1}{2}} \leqslant k\left\|r_{i}^{\prime}\right\|_{L^{2}\left(I_{n}, V\right)},
\end{aligned}
$$

and the fact that $r \in C([0, T], V)$, compare [17, Theorem 3.1]. Hence the first part of the lemma follows by passing to the limit.

In our situation this implies, since $\phi_{t, h}^{\tau} r(t) \in H^{1}\left([0, T], Y_{h}(\tau)\right)$

$$
\begin{aligned}
\left\|\bar{r}^{n}-r(\tau)\right\|_{L^{2}\left(\Gamma^{h}(\tau)\right)}^{2} & =\left\|\frac{1}{k} \int_{I_{n}} \phi_{t, h}^{\tau} r(t)-r(\tau) \mathrm{d} t\right\|_{L^{2}\left(\Gamma^{h}(\tau)\right)}^{2} \leqslant \frac{1}{k} \int_{I_{n}}\left\|\phi_{t, h^{\tau}}^{\tau} r(t)-r(\tau)\right\|_{L^{2}\left(\Gamma^{h}(\tau)\right)}^{2} \mathrm{~d} t \\
& \leqslant k \int_{I_{n}}\left\|\left(\phi_{t, h}^{\tau} r(t)\right)^{\prime}\right\|_{L^{2}\left(\Gamma^{h}(\tau)\right)}^{2} \mathrm{~d} t \leqslant k C_{J}^{h} \int_{I_{n}}\|\dot{r}\|_{L^{2}\left(\Gamma^{h}(t)\right)}^{2} \mathrm{~d} t
\end{aligned}
$$

This proves 1 ., in order to get 2 . integrate over $I_{n}$.

We are now prepared to prove the main result of this section.

THEOREM 6.6 Let $f \in L_{L^{2}(\Gamma)}^{2}$, and let $y_{h}$ and $y_{h, k}$ solve (5.7) and (6.2), respectively, with $y_{0}^{h} \in$ $L^{2}\left(\Gamma_{0}^{h}\right)$ and $f_{h}=f_{l}$. There exists a constant $C>0$ independent of $h, k>0$ and of $f$ and $y_{0}^{h}$ such that

$$
\left\|y_{h}-y_{h, k}\right\|_{L_{L^{2}\left(\Gamma^{h}\right)}^{2}} \leqslant C k\left(\left\|\dot{y}_{h}\right\|_{\left.L_{L^{2}(\Gamma}^{2}\right)}+\|f\|_{L_{L^{2}(\Gamma)}^{2}}+\left\|y_{0}^{h}\right\|_{L^{2}\left(\Gamma_{0}^{h}\right)}\right) .
$$

Proof. The proof is inspired by [23, Theorem 5.2], compare also [26, Theorem 1.2.5] and [20, Theorem 5.1]. Test (5.7) with $\phi_{t_{n}, h}^{t} \varphi, \varphi \in Y_{h}$ and integrate over $I_{n}$ to obtain

$$
\left\langle y_{h}\left(t_{n}\right), \varphi\right\rangle_{n}-\left\langle y_{h}\left(t_{n-1}\right), \varphi\right\rangle_{n-1}+\int_{[0, T]} \mathfrak{a}\left(t ; y_{h}, \varphi\right) \mathrm{d} t=\int_{[0, T]}\left\langle f_{l}, \varphi\right\rangle_{L^{2}\left(\Gamma^{h}(t)\right)} \mathrm{d} t
$$


Solve the adjoint equation (6.4) for $z$ with both right-hand side and test function $\varphi=g=$ $\sum_{n=1}^{N}\left(\bar{y}_{h}^{n}-y_{h, k}^{n}\right) \mathbf{1}_{I_{n}}$

$$
\int_{I_{n}}\left\|\bar{y}_{h}^{n}-y_{h, k}^{n}\right\|_{n}^{2} \mathrm{~d} t=\left\langle z^{n}-z^{n+1}, \bar{y}_{h}^{n}-y_{h, k}^{n}\right\rangle_{n}+k \mathfrak{a}_{n}\left(\bar{y}_{h}^{n}-y_{h, k}^{n}, z^{n}\right)
$$

Subtract (6.9) from (6.2). Tested with $z$ this yields

$$
\begin{aligned}
\left\langle y_{h, k}^{n}-y_{h}\left(t_{n}\right), z^{n}\right\rangle_{n} & -\left\langle y_{h, k}^{n-1}-y_{h}\left(t_{n-1}\right), z^{n}\right\rangle_{n-1}+k \mathfrak{a}_{n}\left(y_{h, k}^{n}-\bar{y}_{h}^{n}, z^{n}\right) \\
= & \int_{I_{n}} \mathfrak{a}\left(t ; y_{h}, z^{n}\right) \mathrm{d} t-k \mathfrak{a}_{n}\left(\bar{y}_{h}^{n}, z^{n}\right)+k\left\langle\bar{f}_{l}^{n}, z^{n}\right\rangle_{n}-\int_{I_{n}}\left\langle f_{l}, z^{n}\right\rangle_{L^{2}\left(\Gamma^{h}(t)\right)} \mathrm{d} t
\end{aligned}
$$

Let $\bar{y}_{h}=\sum_{n=1}^{N} \bar{y}_{h}^{n} \mathbf{1}_{I_{n}}$. Add (6.10) and sum up over $1 \leqslant n \leqslant N$ to get

$$
\begin{aligned}
& \left\langle f_{l}, z\right\rangle_{h, k}-\left\langle f_{l}, z\right\rangle_{L_{L^{2}\left(\Gamma^{h}\right)}^{2}}+\sum_{n=1}^{N} \int_{I_{n}}\left\|\bar{y}_{h}-y_{h, k}\right\|_{n}^{2} \mathrm{~d} t+\int_{I_{n}} \mathfrak{a}\left(t ; y_{h}, z^{n}\right) \mathrm{d} t-k \mathfrak{a}\left(t_{n} ; \bar{y}_{h}^{n}, z^{n}\right) \\
& =\sum_{n=1}^{N}\left[k \mathfrak{r}_{n}\left(\bar{y}_{h}^{n}, z^{n}\right)+\left\langle\bar{y}_{h}^{n}-y_{h}\left(t_{n}\right), z^{n}\right\rangle_{n}-\left\langle y_{h, k}^{n-1}-y_{h}\left(t_{n-1}\right), z^{n}\right\rangle_{n-1}-\left\langle z^{n+1}, \bar{y}_{h}^{n}-y_{h, k}^{n}\right\rangle_{n}\right] \\
& =\left\langle y_{h, k}^{N}-y_{h}\left(t_{N}\right), z^{N+1}\right\rangle_{N}-\left\langle y_{h, k}^{0}-y_{h}\left(t_{0}\right), z^{1}\right\rangle_{0}+\sum_{n=1}^{N} k \mathfrak{r}_{n}\left(\bar{y}_{h}^{n}, z^{n}\right) \\
& \quad+\left\langle\bar{y}_{h}^{n}-y_{h}\left(t_{n}\right), z^{n}-z^{n+1}\right\rangle_{n} \\
& =\sum_{n=1}^{N} k \mathfrak{r}_{n}\left(\bar{y}_{h}^{n}, z^{n}\right)+\left\langle\bar{y}_{h}^{n}-y_{h}\left(t_{n}\right), z^{n}-z^{n+1}\right\rangle_{n},
\end{aligned}
$$

and finally, bringing to bear everything we have, i.e. the estimates from Lemma 6.3 for $\mathfrak{a}$, from Lemma 6.2 for the $L^{2}$-norms, and the bound on $\mathfrak{r}$ from (6.3), we arrive at

$$
\begin{aligned}
& \sum_{n=1}^{N} \int_{I_{n}}\left\|\bar{y}_{h}-y_{h, k}\right\|_{n}^{2} \mathrm{~d} t \leqslant\left(k \sum_{n=1}^{N}\left\|\bar{y}_{h}^{n}-y_{h}\left(t_{n}\right)\right\|_{n}^{2}\right)^{\frac{1}{2}}\left(\frac{1}{k} \sum_{n=1}^{N}\left\|z^{n}-z^{n+1}\right\|_{n}^{2}\right)^{\frac{1}{2}} \\
& +C\left(k \sum_{n=1}^{N}\left(\int_{I_{n}}\left\|\phi_{t, h}^{t_{n}} y_{h}\right\|_{Y_{h}\left(t_{n}\right)} \mathrm{d} t\right)^{2}\right)^{\frac{1}{2}}\left(k \sum_{n=1}^{N}\left\|z^{n}\right\|_{Y_{h}\left(t_{n}\right)}^{2}\right)^{\frac{1}{2}}+C k\|f\|_{L_{L^{2}(\Gamma)}^{2}} \underbrace{\left\|z^{l}\right\|_{L_{L^{2}(\Gamma)}^{2}}}_{\leqslant C\|z\|_{h, k}} .
\end{aligned}
$$

Hence using Lemma 6.4 on $z$ we can divide by $\left\|\bar{y}_{h}-y_{h, k}\right\|_{h, k}$. The Lemmas 6.2 and 6.3 allow us to estimate the involved norms, and because of the stability of the space discretization, compare Lemma 5.9, we can estimate the $Y_{h}(t)$-term, to finally arrive at

$$
\left\|\bar{y}_{h}-y_{h, k}\right\|_{L_{L^{2}\left(\Gamma^{2}\right)}} \leqslant C\left(\left(k \sum_{n=1}^{N}\left\|\bar{y}_{h}^{n}-y_{h}\left(t_{n}\right)\right\|_{n}^{2}\right)^{\frac{1}{2}}+k\|f\|_{L_{L^{2}(\Gamma)}^{2}}+k\left\|y_{0}^{h}\right\|_{L^{2}\left(\Gamma_{0}^{h}\right)}\right) .
$$


We now apply Lemma 6.5[2.] to the error $e_{k}=y_{h, k}-y_{h}$ and the averaged error $\bar{e}_{k}=y_{h, k}-\bar{y}_{h}$ and sum up to obtain $\left\|e_{k}-\bar{e}_{k}\right\|_{L_{L^{2}\left(\Gamma^{h}\right)}^{2}} \leqslant C k\left\|\dot{y}_{h}\right\|_{L_{L^{2}\left(\Gamma^{h}\right)}^{2}}$. Combine with (6.11) and 6.5[1.] to estimate

$$
\begin{aligned}
\left\|e_{k}\right\|_{L_{L^{2}\left(\Gamma^{h}\right)}^{2}} \leqslant C k\left\|\dot{y}_{h}\right\|_{L_{L^{2}\left(\Gamma^{h}\right)}^{2}} & +\left\|\bar{e}_{k}\right\|_{L_{L^{2}\left(\Gamma^{h}\right)}^{2}} \\
& \leqslant C k\left(\left\|\dot{y}_{h}\right\|_{L_{L^{2}\left(\Gamma^{h}\right)}^{2}}+\|f\|_{L_{L^{2}(\Gamma)}^{2}}+\left\|y_{0}^{h}\right\|_{L^{2}\left(\Gamma_{0}^{h}\right)}\right) .
\end{aligned}
$$

With view of the stability assertions from (3.10) and Lemma 5.9 and together with Theorem 5.12 we get the following Corollary.

COROLlary 6.7 In the situation of Theorem 6.6 let in addition $\lambda=\mu_{l}$ and $y_{0} \in H^{2}\left(\Gamma_{0}\right)$, and choose $y_{0}^{h}$ as the piecewise linear interpolation of $\left(y_{0}\right)_{l}$. There exists a constant $C>0$ independent of $h, k>0$ and of $f$ and $y_{0}$ such that

$$
\left\|y_{h, k}^{l}-y\right\|_{L_{L^{2}(\Gamma)}^{2}} \leqslant C\left(h^{2}+k\right)\left(\left\|y_{0}\right\|_{H^{2}\left(\Gamma_{0}\right)}+\|f\|_{L_{L^{2}(\Gamma)}^{2}}\right) .
$$

As addressed in Remark 5.13, it should be possible to relax the condition on $y_{0}$ into $y_{0} \in$ $H^{1}\left(\Gamma_{0}\right)$ using the $L^{2}\left(\Gamma_{0}\right)$-projection or the $L^{2}\left(\Gamma_{0}^{h}\right)$-projection $P_{0}^{h}$.

But even in the case of low regularity we still get a uniform estimate.

COROLlary 6.8 In the situation of Theorem 6.6 let only $y_{0} \in L^{2}\left(\Gamma_{0}\right)$ hold while $f \equiv 0$. Let further $y_{0}^{h}=P_{0}^{h}\left(\left(y_{0}\right)_{l}\right)$. There exists a constant $C>0$ independent of $h, k>0$ and of $y_{0}$ such that

$$
\left\|y_{h, k}^{l}-y\right\|_{L_{L^{2}(\Gamma)}^{2}} \leqslant C\left(h+\sup _{t \in[0, T]}\left\|\lambda^{l}-\mu\right\|_{L^{\infty}(\Gamma(t))}+\frac{k}{h}\right)\left\|y_{0}\right\|_{L^{2}\left(\Gamma_{0}\right)} .
$$

Proof. Regarding Theorem 5.14 and 6.6 it remains to bound $\left\|\dot{y}_{h}\right\|_{L_{L^{2}\left(\Gamma^{h}\right)}}$. Like in the proof of Theorem 5.14, using Lemma 5.9 and an inverse estimate, we arrive at the desired estimate.

In particular, for $\kappa>0$, choose $k=\kappa h^{2}$ and $\lambda$ such that $\sup _{t \in[0, T]}\left\|\lambda^{l}-\mu\right\|_{L^{\infty}(\Gamma(t))} \leqslant C h$ to get an $\mathcal{O}(h)$-convergent scheme.

REMARK 6.9 Note that our freedom in the choice of $\mathfrak{r}$ now allows us to finally drop the conditions on $\lambda$ and $\mu$, respectively, in (5.6), (5.7), and (6.1). Let us assume we want to approximate the solution $y$ of (5.6) with $\mu \equiv 0, y_{0} \in H^{1}(\Gamma(0))$, and $f \in L_{L^{2}(\Gamma)}^{2}$. Now $y_{h, k} \in W_{k}^{h}$ solves

$$
\begin{aligned}
& y_{h, k}^{0}= y_{0}^{h}, \quad \forall \varphi \in W_{k}^{h}, \quad 1 \leqslant n \leqslant N: \\
&\left\langle y_{h, k}^{n}, \varphi\right\rangle_{n}-\left\langle y_{h, k}^{n-1}, \varphi\right\rangle_{n-1}+k \int_{\Gamma^{h}\left(t_{n}\right)} \nabla_{\Gamma^{h}\left(t_{n}\right)} y_{h, k}^{n} \cdot \nabla_{\Gamma^{h}\left(t_{n}\right)} \varphi \mathrm{d} \Gamma^{h}\left(t_{n}\right)=k\left\langle\bar{f}_{h}^{n}, \varphi\right\rangle_{n},
\end{aligned}
$$




$$
\begin{aligned}
& \text { iff } y_{h, m, \lambda}=\sum_{n=1}^{N} e^{-\lambda t_{n}} y_{h, k}^{n} \mathbf{1}_{I_{n}} \in W_{k}^{h}, \lambda>0 \text { solves } \\
& \begin{aligned}
y_{h, k, \lambda}^{0}=y_{0}^{h}, \quad \forall \varphi \in W_{k}^{h}, \quad 1 \leqslant n \leqslant N: \\
\left\langle y_{h, k, \lambda}^{n}, \varphi\right\rangle_{n}-\left\langle y_{h, k, \lambda}^{n-1}, \varphi\right\rangle_{n-1}+k \int_{\Gamma^{h}\left(t_{n}\right)} \nabla_{\Gamma^{h}\left(t_{n}\right)} y_{h, k, \lambda}^{n} \cdot \nabla_{\Gamma^{h}\left(t_{n}\right)} \varphi+\lambda y_{h, k, \lambda}^{n} \varphi \mathrm{d} \Gamma^{h}\left(t_{n}\right) \\
\quad=k\left\langle e^{\left.-\lambda t_{n-1} \bar{f}_{h}^{n}, \varphi\right\rangle_{n},}\right.
\end{aligned}
\end{aligned}
$$

with

$$
k \mathfrak{r}_{n}(\psi, \varphi)=\left(e^{\lambda k}-1-\lambda k\right)\langle\psi, \varphi\rangle_{n}+k\left(e^{\lambda k}-1\right) \int_{\Gamma^{h}\left(t_{n}\right)} \nabla_{\Gamma^{h}\left(t_{n}\right)} \psi \cdot \nabla_{\Gamma^{h}\left(t_{n}\right)} \varphi \mathrm{d} \Gamma^{h}\left(t_{n}\right) .
$$

Taking into account that $\left\|e^{-\lambda t} f(t)-\sum_{n=1}^{N} e^{-\lambda t_{n}} \mathbf{1}_{I_{n}} f(t)\right\|_{L_{L^{2}(\Gamma)}^{2}} \leqslant k\|f\|_{L_{L^{2}(\Gamma)}^{2}}$, we apply Corollary 6.7 to $y_{h, m, \lambda}$ and conclude $\left\|y_{h, k}^{l}-y\right\|_{L_{L^{2}(\Gamma)}^{2}} \leqslant C e^{\lambda T}\left(h^{2}+k\right)$.

\section{Variational discretization}

We now return to problem $\left(\mathbb{P}_{d}\right)$ which has the advantage over $\left(\mathbb{P}_{T}\right)$, that its adjoint equation satisfies the regularity estimate (3.10). For $\left(\mathbb{P}_{T}\right)$ this is not the case iff $y_{T} \in L^{2}(\Gamma(T)) \backslash H^{1}(\Gamma(T))$. In the spirit of [12], let us approximate $\left(\mathbb{P}_{d}\right)$ by

$$
\left(\mathbb{P}_{d}^{h}\right) \quad\left\{\begin{array}{l}
\min _{u \in L_{L^{2}\left(\Gamma^{h}\right)}^{2}} O(u):=\frac{1}{2}\left\|S_{d}^{h}(u)-\left(y_{d}\right)_{l}\right\|_{h, k}^{2}+\frac{\alpha}{2}\|u\|_{h, k}^{2} \\
\text { s.t. } a \leqslant u \leqslant b
\end{array}\right.
$$

with $\left\{\Gamma^{h}(t)\right\}_{t \in[0, T]}$ as in Section 5 and $S_{d}^{h}:\left(L_{L^{2}\left(\Gamma^{h}\right)}^{2},\langle\cdot, \cdot\rangle_{h, k}\right) \rightarrow\left(L_{L^{2}\left(\Gamma^{h}\right)}^{2},\langle\cdot, \cdot\rangle_{h, k}\right), f_{h} \mapsto$ $y_{f}$ is defined through the scheme 6.2 with $\lambda \equiv 0$ and $y_{0}^{h} \equiv 0$. We choose the scalar product $\langle\cdot, \cdot\rangle_{h, k}$ defined in (6.5) in order to obtain a computable scheme to evaluate $S_{d}^{h^{*}}$, namely (6.4) with $z^{N+1}=0$. Given snapshots $\left\{\Gamma^{h}\left(t_{n}\right)\right\}_{n=1}^{N}$, the product $\langle\cdot, \cdot\rangle_{h, k}$ can be evaluated exactly for functions $\varphi_{h} \in W_{k}^{h}$ as well as for $P_{[a, b]}\left(\varphi_{h}\right)$.

Let $U_{\mathrm{ad}}^{h}=\left\{v \in L_{L^{2}\left(\Gamma^{h}\right)}^{2} \mid a \leqslant v \leqslant b\right\}$. As in (4.2) the first order necessary optimality condition for an optimum $u_{h}$ of $\left(\mathbb{P}_{d}^{h}\right)$ is

$$
\left\langle\alpha u_{h}+S_{d}^{h^{*}}\left(S_{d}^{h} u_{h}-\left(y_{d}\right)_{l}\right), v-u_{h}\right\rangle_{h, k} \geqslant 0, \quad \forall v \in U_{\text {ad }} .
$$

First note that as in the continuous case the $\langle\cdot, \cdot\rangle_{h, k}$-orthogonal projection onto $U_{\text {ad }}^{h}$ coincides with the point-wise projection $P_{[a, b]}(v)$. Similar to 4.4 we get

$$
u_{h}=P_{[a, b]}\left(-\frac{1}{\alpha} p_{d}^{h}(u)\right), \quad p_{d}^{h}(u)=S_{d}^{h^{*}}\left(S_{d}^{h} u-\left(y_{d}\right)_{l}\right) .
$$

Equation (7.2) is amenable to a semi-smooth Newton method that, while still being implementable, operates entirely in $L_{L^{2}\left(\Gamma^{h}\right)}^{2}$. The implementation requires one to resolve the boundary between the 
inactive set $\ell_{u}\left(t_{n}\right)=\left\{\gamma \in \Gamma\left(t_{n}\right) \mid a<-\frac{1}{\alpha} p_{d}^{h}(u)[\gamma]<b\right\}$ and the active set $Q_{u}\left(t_{n}\right)=\Gamma^{h}\left(t_{n}\right) \backslash$ $\ell_{u}\left(t_{n}\right)$ for $1 \leqslant n \leqslant N$. For details on the implementation see [14] and [15]. Note that in order to implement $S_{d}^{h}$ and $S_{d}^{h^{*}}$ according to (6.2) and (6.4) for right-hand sides in $W_{k}^{h}$, again one only needs to know the snapshots $\left\{\Gamma^{h}\left(t_{n}\right)\right\}_{n=0}^{N}$. The solution of $\left(\mathbb{P}_{d}^{h}\right)$ converges towards that of $\left(\mathbb{P}_{d}\right)$ and the order of convergence is optimal in the sense that it is given by the order of convergence of $S_{d}^{h}$ and $S_{d}^{h^{*}}$.

THEOREM 7.1 (Order of Convergence for $\left(\mathbb{P}_{d}^{h}\right)$ ) Let $u \in L_{L^{2}(\Gamma)}^{2}, u_{h} \in L_{L^{2}\left(\Gamma^{h}\right)}^{2}$ be the solutions of $\left(\mathbb{P}_{d}\right)$ and $\left(\mathbb{P}_{d}^{h}\right)$, respectively. Let $C>1$. Then for sufficiently small $h, k>0$ there holds

$$
\begin{aligned}
& 2 \alpha\left\|u_{h}^{l}-u\right\|_{L_{L^{2}(\Gamma)}^{2}}^{2}+\left\|y_{h}^{l}-y\right\|_{L_{L^{2}(\Gamma)}^{2}}^{2} \\
& \quad \leqslant C\left(2\left(\left((\cdot)^{l} S_{d}^{h^{*}}(\cdot)_{l}-S_{d}^{*}\right)\left(y-y_{d}\right), u-u_{h}^{l}\right\rangle_{L_{L^{2}(\Gamma)}^{2}}+\left\|\left((\cdot)^{l} S_{d}^{h}(\cdot)_{l}-S_{d}\right) u\right\|_{L_{L^{2}(\Gamma)}^{2}}^{2}\right),
\end{aligned}
$$

with $y=S_{d} u$ and $y_{h}=S_{d}^{h} u_{h}$.

Proof. Let $\mathrm{P}_{U_{\mathrm{ad}}^{h}}(\cdot)$ denote the $\langle\cdot, \cdot\rangle_{h, k}$-orthogonal projection onto $U_{\mathrm{ad}}^{h}$. We have

$$
u_{l}=P_{[a, b]}\left(-\frac{1}{\alpha} p_{d}(u)\right)_{l}=P_{[a, b]}\left(-\frac{1}{\alpha} p_{d}(u)_{l}\right)=\mathrm{P}_{U_{\mathrm{ad}}^{h}}\left(-\frac{1}{\alpha} p_{d}(u)_{l}\right) .
$$

Since $u_{h}, u_{l} \in U_{\text {ad }}^{h}$ we can plug $u_{h}$ into the variational inequality for $\mathrm{P}_{U_{a d}^{h}}(\cdot)$ and $u_{l}$ into the optimality condition (7.1)

$$
\left\langle-\frac{1}{\alpha} p_{d}(u)_{l}-u_{l}, u_{h}-u_{l}\right\rangle_{h, k} \leqslant 0, \quad\left\langle\alpha u_{h}+p_{d}^{h}\left(u_{h}\right), u_{l}-u_{h}\right\rangle_{h, k} \geqslant 0 .
$$

From here the proof is a standard task, compare [13, Theorem 3.4] and [14].

For the problem

$$
\left(\mathbb{P}_{T}^{h}\right) \quad\left\{\begin{array}{l}
\min _{u \in L_{L^{2}\left(\Gamma^{h}\right)}^{2}} \mathcal{O}(u):=\frac{1}{2}\left\|S_{T}^{h}(u)-\left(y_{T}\right)_{l}\right\|_{L^{2}\left(\Gamma^{h}(T)\right)}^{2}+\frac{\alpha}{2}\|u\|_{L_{L^{2}\left(\Gamma^{h}\right)}^{2}}^{2} \\
\text { s.t. } a \leqslant u \leqslant b,
\end{array}\right.
$$

one can prove a similar result. Here the operator $S_{T}^{h}$ is the map $f_{h} \rightarrow y_{f}(T)$, according to the scheme (6.2) with $\lambda \equiv 0$.

THEOREM 7.2 (Order of Convergence for $\left(\mathbb{P}_{T}^{h}\right)$ ) Let $u \in L_{L^{2}(\Gamma)}^{2}, u_{h} \in L_{L^{2}\left(\Gamma^{h}\right)}^{2}$ be the solutions of $\left(\mathbb{P}_{T}\right)$ and $\left(\mathbb{P}_{T}^{h}\right)$, respectively. Let $C>1$. Then for sufficiently small $h, k>0$ there holds

$$
\begin{aligned}
& 2 \alpha\left\|u_{h}^{l}-u\right\|_{L_{L^{2}(\Gamma)}^{2}}^{2}+\left\|y_{h}^{l}-y\right\|_{L^{2}(\Gamma(T))}^{2} \\
& \quad \leqslant C\left(2\left\langle\left((\cdot)^{l} S_{T}^{h^{*}}(\cdot)_{l}-S_{T}^{*}\right)\left(y-y_{T}\right), u-u_{h}^{l}\right\rangle_{L_{L^{2}(\Gamma)}^{2}}+\left\|\left((\cdot)^{l} S_{T}^{h}(\cdot)_{l}-S_{T}\right) u\right\|_{L^{2}(\Gamma(T))}^{2}\right),
\end{aligned}
$$

with $y=S_{T} u$ and $y_{h}=S_{T}^{h} u_{h}$. 
Now as to the convergence of $\left((\cdot)^{l} S_{d}^{h^{*}}(\cdot)_{l}-S_{d}^{*}\right)$, note that taking the adjoint does not commute with the discretization. Indeed, apply the scheme (6.2) to the adjoint equation (4.3), i.e. $\lambda=-\left(\operatorname{div} \Gamma\left(t_{n}\right) V\right)_{l}$ to get

$$
\begin{aligned}
& z_{g}^{N+1}=0, \quad \forall \varphi \in W_{k}^{h}, 1 \leqslant n \leqslant N: \\
& \int_{I_{n}}\left\langle\phi_{t, h}^{t_{n}} g_{h}, \varphi\right\rangle_{n} \mathrm{~d} t=\left\langle z_{g}^{n}, \varphi\right\rangle_{n}-\left\langle z_{g}^{n+1}, \varphi\right\rangle_{n} \\
& +k \int_{\Gamma^{h}\left(t_{n}\right)} \nabla_{\Gamma^{h}\left(t_{n}\right)} \varphi \nabla_{\Gamma^{h}\left(t_{n}\right)} z_{g}^{n}-\left(\operatorname{div} \Gamma\left(t_{n}\right) V\right)_{l} \varphi z^{n} \mathrm{~d} \Gamma^{h}\left(t_{n}\right)+\int_{I_{n}}\left\langle\varphi \operatorname{div} \Gamma^{h}(t) V_{h}, z^{n}\right\rangle_{L^{2}\left(\Gamma^{h}(t)\right)} \mathrm{d} t,
\end{aligned}
$$

instead of (6.4).

In the situation of $\left(\mathbb{P}_{d}^{h}\right)$ however, this discrepancy can be remedied by Lemma 5.7 which implies

$$
\left\|(\cdot)_{l}-(\cdot)^{l^{*}}\right\|_{\mathcal{L}\left(L_{L^{2}(\Gamma)}^{2}, L_{L^{2}(\Gamma h)}^{2}\right)},\left\|(\cdot)^{l}-(\cdot)_{l}^{*}\right\|_{\mathcal{L}\left(L_{L^{2}\left(\Gamma^{h}\right)}^{2}, L_{L^{2}(\Gamma)}^{2}\right)} \leqslant C h^{2},
$$

and due to Lemma 6.2 which allows us to conclude

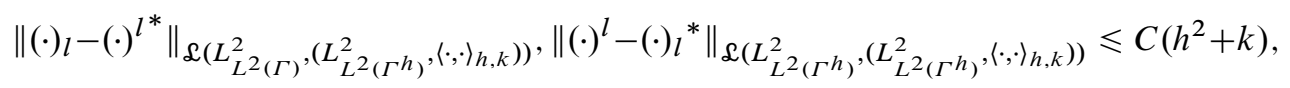

if we interpret $(\cdot)_{l},(\cdot)^{l}$ as operators into or on $\left(L_{L^{2}\left(\Gamma^{h}\right)}^{2},\langle\cdot, \cdot\rangle_{h, k}\right)$, respectively.

Hence we get the estimate

$$
\begin{aligned}
\left\|(\cdot)^{l} S_{d}^{h^{*}}(\cdot)_{l}-S_{d}^{*}\right\| & \leqslant\left\|\left((\cdot)^{l}-(\cdot)_{l}{ }^{*}\right) S_{d}^{h^{*}}(\cdot)_{l}\right\|+\left\|(\cdot)_{l}{ }^{*} S_{d}^{h^{*}}\left((\cdot)_{l}-(\cdot)^{l^{*}}\right)\right\|+\left\|(\cdot)_{l}{ }^{*} S_{d}^{h^{*}}(\cdot)^{l^{*}}-S_{d}^{*}\right\| \\
& \leqslant C\left(k+h^{2}\right),
\end{aligned}
$$

in the $\mathcal{L}\left(L_{L^{2}(\Gamma)}^{2}, L_{L^{2}(\Gamma)}^{2}\right)$-operator norm.

As opposed to problem $\left(\mathbb{P}_{d}^{h}\right)$, in the case of $\left(\mathbb{P}_{T}\right)$ it is easier to proof the convergence of $S_{T}^{h *}$ than that of $S_{T}^{h}$ itself. In the sense of (6.2), consider the discretization of the adjoint operator $S_{T}^{*}$

$$
S_{T}^{h^{*}}: L^{2}\left(\Gamma^{h}(T)\right) \ni z_{T} \mapsto z \in W_{k}^{h} \subset\left(L_{L^{2}\left(\Gamma^{h}\right)}^{2},\langle\cdot, \cdot\rangle_{h, k}\right)
$$

according to the primal scheme after the time-transform $t^{\prime}=T-t$

$$
\begin{aligned}
& z^{N+1}=z_{T}, \quad \forall \varphi \in W_{k}^{h}, \quad 1 \leqslant n \leqslant N: \\
& \left\langle z^{n}, \varphi^{n}\right\rangle_{n}-\left\langle z^{n+1}, \varphi^{n}\right\rangle_{n+1}+k \int_{\Gamma^{h}\left(t_{n}\right)} \nabla_{\Gamma^{h}\left(t_{n}\right)} z^{n} \nabla_{\Gamma^{h}\left(t_{n}\right)} \varphi^{n}+\operatorname{div} \Gamma^{h}\left(t_{n}\right) V_{h} z^{n} \varphi^{n} \mathrm{~d} \Gamma^{h}\left(t_{n}\right)=0 .
\end{aligned}
$$

Corollary 6.8 applies an yields $\left\|(\cdot)^{l} S_{T}^{h^{*}}(\cdot)_{l}-S_{T}^{*}\right\|_{\mathcal{L}\left(L^{2}(\Gamma(T)), L_{L^{2}(\Gamma)}^{2}\right)} \leqslant C\left(h+\frac{k}{h}\right)$.

Now in addition to (7.3) we have just like in the time-dependent case

$$
\left\|(\cdot)_{l}-(\cdot)^{l^{*}}\right\|_{\mathcal{L}\left(L^{2}(\Gamma(T)), L^{2}\left(\Gamma^{h}(T)\right)\right)} \leqslant C h^{2},
$$



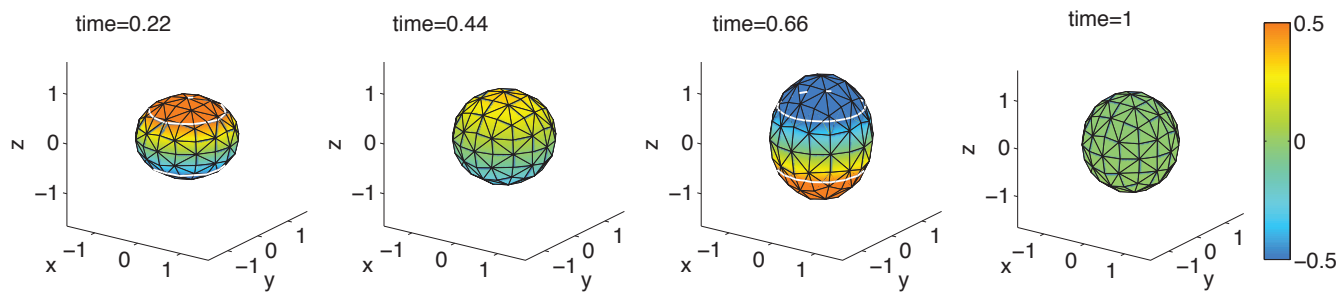

FIG. 1. Selected time snapshots of $\bar{u}_{h}$ computed for Example 8.1 on the Sphere after 4 refinements

compare [3, Lemma 5.1]. We conclude

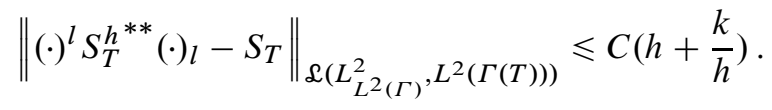

Hence, the operator $\left.S_{T}^{h}=S_{T}^{h^{* *}}:\left(L_{L^{2}\left(\Gamma^{h}\right)}^{2},\langle\cdot, \cdot\rangle_{h, k}\right) \rightarrow L^{2}\left(\Gamma^{h}(T)\right)\right)$ is a discretization of $S_{T}$. Also, the mapping $S_{T}^{h}: u_{h} \mapsto y_{h, k}(T)$ is implemented by the scheme

$$
\begin{aligned}
& y^{0}=0, \quad \forall \varphi \in W_{k}^{h}, \quad 1 \leqslant n \leqslant N: \\
& \left\langle y^{n}, \varphi^{n}\right\rangle_{n}-\left\langle y^{n-1}, \varphi^{n}\right\rangle_{n} \\
& \quad+k \int_{\Gamma^{h}\left(t_{n}\right)} \nabla_{\Gamma^{h}\left(t_{n}\right)} y^{n} \nabla_{\Gamma^{h}\left(t_{n}\right)} \varphi^{n}+\left(\operatorname{div}_{\Gamma^{h}\left(t_{n}\right)} V_{h}\right)_{l} y^{n} \varphi^{n} \mathrm{~d} \Gamma^{h}\left(t_{n}\right)=k\left\langle\bar{u}_{h}^{n}, \varphi^{n}\right\rangle_{n},
\end{aligned}
$$

as shows summation over $1 \leqslant n \leqslant N$.

If $y_{T}$ is more regular, such as $y_{T} \in H^{1}(\Gamma(T))$, then one might want to apply results from [5] that state $h^{2}$-convergence of the discretization $S_{T}^{h}$, yet not in the $\mathcal{L}\left(L_{L^{2}(\Gamma)}^{2}, L^{2}(\Gamma(T))\right)$-norm. In order to to so, it remains to ensure the regularity assumptions of [5, Theorem 4.4] to be met by the optimal control $u$.

\section{Example}

Provided the results from [11] and [25] hold on surfaces, Equation (7.2) is semi-smooth due to the smoothing properties of $S_{d}^{h *}$, i.e. the stability ensured by Lemma 6.4. The lemma a priori holds only in the case $\lambda \geqslant 1$, but can be extended for arbitrary $\lambda, \mu$ by rescaling, see Remark 6.9. By Lemma 6.4 the operator $\phi_{\cdot, h}^{s} S_{d}^{h^{*}}$ continuously maps $\left(L_{L^{2}\left(\Gamma^{h}\right)}^{2},\langle\cdot, \cdot\rangle_{h, k}\right)$ into

$$
L^{\infty}\left([0, T], Y_{h}(s)\right) \subset L^{p}\left([0, T], L^{p}\left(\Gamma^{h}(s)\right)\right) \simeq L^{p}\left([0, T] \times \Gamma^{h}(s)\right)
$$

for every $2<p<\infty$. This would imply semi-smoothness of the operator

$$
P_{[a, b]}\left(-\frac{1}{\alpha} \phi_{t, h}^{s}\left(p_{d}^{h}\left(\phi_{s, h}^{t}(\cdot)\right)\right)\right): L^{2}\left([0, T] \times \Gamma^{h}(s)\right) \rightarrow L^{2}\left([0, T] \times \Gamma^{h}(s)\right),
$$

compare [25], and thus of equation (7.2).

We implemented a semi-smooth Newton Algorithm for (7.2), along the lines of [15]. 
PARABOLIC OPTIMAL CONTROL PROBLEMS ON EVOLVING SURFACES

TABLE 1. $L^{2}$-error, $L^{\infty}$-error and the corresponding EOCs for Example 8.1

\begin{tabular}{cccccccccc}
\hline $\mathrm{R}$ & $E R R_{L^{2}}$ & $E O C_{L^{2}}$ & $E R R_{\infty}$ & $E O C_{\infty}$ & $\mathrm{R}$ & $E R R_{L^{2}}$ & $E O C_{L^{2}}$ & $E R R_{\infty}$ & $E O C_{\infty}$ \\
\hline 0 & $1.68 \mathrm{e}-01$ & - & $8.71 \mathrm{e}-01$ & - & 5 & $6.78 \mathrm{e}-03$ & 2.15 & $1.08 \mathrm{e}-01$ & 2.01 \\
1 & $5.40 \mathrm{e}-02$ & - & $7.88 \mathrm{e}-01$ & - & 6 & $3.15 \mathrm{e}-03$ & 2.09 & $5.01 \mathrm{e}-02$ & 1.97 \\
2 & $4.13 \mathrm{e}-02$ & 2.45 & $5.32 \mathrm{e}-01$ & 0.86 & 7 & $1.72 \mathrm{e}-03$ & 2.03 & $2.80 \mathrm{e}-02$ & 1.99 \\
3 & $2.60 \mathrm{e}-02$ & 1.78 & $3.78 \mathrm{e}-01$ & 1.79 & 8 & $7.92 \mathrm{e}-04$ & 2.02 & $1.31 \mathrm{e}-02$ & 1.97 \\
4 & $1.24 \mathrm{e}-02$ & 2.21 & $1.82 \mathrm{e}-01$ & 1.97 & & & & & \\
\hline
\end{tabular}

EXAMPLE 8.1 (High Regularity) Consider problem $\left(\mathbb{P}_{d}\right)$ with $\alpha=1, a=-\frac{1}{2}, b=\frac{1}{2}, T=1$, and $\Gamma_{0} \subset \mathbb{R}^{3}$ the unit sphere. Let $\Gamma(t)=\bar{\Phi}_{0}^{t} \Gamma_{0}$ with $\bar{\Phi}_{0}^{t}(x, y, z)=(x, y, z / \rho(t))^{T}$ and $\rho(t)=e^{\frac{\sin (2 \pi t)}{4}}$. In coordinates $(x, y, z)$ of $\mathbb{R}^{3}$ let $\bar{u}=P_{\left[-\frac{1}{2}, \frac{1}{2}\right]}(z \sin (2 \pi t))$ and $y_{d}=\tilde{y}_{d}+S_{d} \bar{u}$ with

$$
\tilde{y}_{d}=-\alpha\left(\left(\frac{\pi}{2} \sin (2 \pi t)-2 \pi\right) \cos (2 \pi t)+\frac{\sin (2 \pi t) \rho^{2}}{x^{2}+y^{2}+\rho^{4} z^{2}}\left(\rho^{2}+1-z^{2} \frac{\rho^{6}-\rho^{4}}{x^{2}+y^{2}+\rho^{4} z^{2}}\right)\right) z \text {. }
$$

Then $\bar{u}$ solves $\left(\mathbb{P}_{d}\right)$.

In order to compute the solution $\bar{u}_{h}$ of $\left(\mathbb{P}_{d}^{h}\right)$ we construct triangulations of $\Gamma_{0}$ from our macrotriangulation $R_{0}$, i.e. the cube whose nodes reside on $\Gamma_{0}$ triangulated into 12 rectangular triangles. We generate $R_{i+1}$ from $R_{i}$ through longest edge refinement followed by projecting the inserted vertices onto $\Gamma_{0}$.

Table 1 shows the relative error in the $L_{L^{2}\left(\Gamma^{h}\right)}^{2}$-norm and the relative $L^{\infty}$-error

$$
E R R_{\infty}=\frac{\left\|\phi_{t, h}^{s}\left(\bar{u}_{h}-\bar{u}_{l}\right)\right\|_{L^{\infty}\left([0, T] \times \Gamma^{h}(s)\right)}}{\left\|\phi_{t, h}^{s} \bar{u}_{l}\right\|_{L^{\infty}\left([0, T] \times \Gamma^{h}(s)\right)}},
$$

as well as the corresponding experimental orders of convergence

$$
E O C_{i}=\ln \frac{E R R_{i}}{E R R_{i-q}}\left(\ln \frac{H_{i}}{H_{i-q}}\right)^{-1}
$$

where $H$ denotes the maximal edge length of $\Gamma_{0}^{h}$, see Table 2. Throughout this section we chose $q=2$ for both $E O C_{L^{2}}$ and $E O C_{L} \infty$, and the time step length is $k=\frac{1}{20} H^{2}$.

Figure 1 shows the solution of $\left(\mathbb{P}_{d}^{h}\right)$ at different points in time. Note that the white line marks the border between active and inactive sets. On the active parts, the optimal control assumes the value $a$ or $b$, respectively.

Let us conclude with an example for $\left(\mathbb{P}_{T}^{h}\right)$ with a desired state $y_{T}$ that just barely lies in $L^{2}(\Gamma(T))$. In this situation we can only expect $\Theta(h)$-convergence. We consider the unconstrained problem

EXAMPLE 8.2 (Low Regularity) Consider problem $\left(\mathbb{P}_{T}\right)$ with $\alpha=1, a=-\infty, b=\infty, T=1$ and $\Gamma(t)$ as in Example 8.1. Let $y_{T}=\frac{1}{(x+y)^{0.49}}$.

Since we do not know the exact solution of Example 8.2, we estimate the relative error by $E R R_{L^{2}}^{i} \simeq\left\|\bar{u}_{i}^{l}-\bar{u}_{i+2}\right\|_{L_{L^{2}\left(\Gamma^{i+2}\right)}^{2}} /\left\|\bar{u}_{i+2}\right\|_{L_{L^{2}\left(\Gamma^{i}+2\right)}^{2}}$, where $\bar{u}_{i}$ denotes the solution of $\left(\mathbb{P}_{T}^{h}\right)$ on the 
TABLE 2. $L^{2}$-error and the corresponding EOC for Example 8.2. $H$ is the maximal edge length of $\Gamma_{0}^{h}$ (both examples).

\begin{tabular}{rccccccccc}
\hline $\mathrm{R}$ & 1 & 2 & 3 & 4 & 5 & 6 & 7 & 8 & 9 \\
\hline ERR $_{L^{2}}$ & 0.1899 & 0.1444 & 0.1140 & 0.0701 & 0.0484 & 0.0306 & 0.0215 & 0.0147 & 0.0104 \\
EOC $_{L^{2}}$ & - & - & 1.2414 & 1.3272 & 1.3709 & 1.2617 & 1.2030 & 1.0781 & 1.0520 \\
$H$ & 1.1547 & 0.9194 & 0.7654 & 0.5333 & 0.4099 & 0.2769 & 0.2085 & 0.1398 & 0.1047 \\
\hline
\end{tabular}

$i$-th refinement $\left\{\Gamma^{i}(t)\right\}_{t \in[0, T]}$ of $\{\Gamma(t)\}_{t \in[0, T]}$. The lift $(\cdot)^{l}$ is taken perpendicular to the smooth surface $\Gamma(t)$. Table 2 shows the estimated $L^{2}$-errors and corresponding EOCs. We computed the $L^{2}\left(\Gamma^{h}(T)\right)$-projection $P_{T}^{h} y_{T l}$ analytically. Otherwise the error introduced by the numerical integration of the non-smooth function $y_{T}$ would be dominant. It helps that all our triangulations resolve the plane $\{x+y=0\}$.

Acknowledgement. The author would like to thank Prof. Dziuk for the fruitful discussion during his stay in Hamburg in November 2010, and for kindly providing the preprints [4] and [5].

\section{REFERENCES}

1. Coddington, E. A. \& Levinson, N., Theory of ordinary differential equations. McGill-Hill Book Company, 1955. Zbl0064.33002 MR0069338

2. Ciarlet, P. G. \& Lions, J. L., editors, Handbook of numerical analysis. Volume II: Finite element methods (Part 1). North-Holland, 1991. Zbl0712.65091

3. Dziuk, G. \& Elliott, C.M., Finite elements on evolving surfaces. IMA J. Numer. Anal., 27 (2007), 262-292. Zbl1120.65102 MR2317005

4. Dziuk, G. \& Elliott, C. M., L2-estimates for the evolving surface finite element method, Math. Comput. 82 (2013), 1-24. Zbl1269.65087 MR2983013

5. GDziuk, G. \& Elliott, C. M., Fully discrete evolving surface finite element method, SIAM J. Numer. Anal. 50 (2012), 2677-2694. Zbl1267.65128 MR3022237

6. Dziuk, G., Lubich, Ch. \& MAnsour, D., Runge-kutta time discretization of parabolic differential equations on evolving surfaces. IMA J. Numer. Anal. 32 (2012), 394-416. Zb11247.65124 MR2911394

7. Delfour, M. C. \& Zolésio, J.-P. Shapes and geometries. Analysis, differential calculus, and optimization. Philadelphia, PA: SIAM, 2001. Zb11251.49001 MR1855817

8. DzIUK, G., Finite elements for the Beltrami operator on arbitrary surfaces. In Partial differential equations and calculus of variations, number 1357 in Lect. Notes Math., 142-155. Springer, 1988. Zb10663. 65114 MR0976234

9. Eilks, C. \& Elliott, C.M., Numerical simulation of dealloying by surface dissolution via the evolving surface finite element method. J. Comput. Phys. 227 (2008), 9727-9741. Zbl1149. 76027 MR2469030

10. Evans, L. C. Partial differential equations. Number 19 in Graduate Studies in Mathematics. American Mathematical Society (AMS), 1998. Zb10902.35002 MR1625845

11. Hintermüller, M., Ito, K. \& KUnisch, K., The primal-dual active set strategy as a semismooth Newton method. SIAM J. Optim. 13 (2003), 865-888. Zbl1080 . 90074 MR1972219

12. Hinze, M., A variational discretization concept in control constrained optimization: The linear-quadratic case. Comput. Optim. Appl. 30 (2005), 45-61. Zbl1074.65069 MR2122182

13. Hinze, M., Pinnau, R., Ulbrich, M. \& Ulbrich, S., Optimization with PDE constraints. Number 23 in Mathematical Modelling: Theory and Applications. Springer, 2009. Zbl1167.49001 MR2516528 
14. Hinze, M. \& Vierling, M., Optimal control of the Laplace-Beltrami operator on compact surfaces concept and numerical treatment. J. Comput. Math. 30 (2012), 392-403. Zbl1274. 49044 MR2965990

15. Hinze, M. \& Vierling, M., A globalized semi-smooth Newton method for variational discretization of control constrained elliptic optimal control problems. In Constrained Optimization and Optimal Control for Partial Differential Equations. Birkhäuser, 2012. MR3060473

16. LiOnS, J. L., Optimal control of systems governed by partial differential equations. Translated by S.K. Mitter. Number 170 in Die Grundlehren der mathematischen Wissenschaften. Springer, 1971. Zb10203. 09001 MR0271512

17. Lions, J. L. \& Magenes, E., Problèmes aux limites non homogenes et applications. Vol. 1 and 2. Dunod, 1968. Zbl0165.10801 MR0247243 (vol. 1), MR0247244 (vol. 2)

18. Ladyzhenskaya, O. A., Solonnikov, V. A. \& Ural'tseva, N. N., Linear and quasi-linear equations of parabolic type. Translated from the Russian by S. Smith. Number 23 in Translations of Mathematical Monographs. American Mathematical Society (AMS), 1968. Zb10174.15403

19. Martio, O., Miklyukov, V. M. \& Vuorinen, M. Morrey's lemma on Riemannian manifolds. Rev. Roum. Math. Pures Appl. 43 (1998), 183-210. Zbl0928.46017 MR1655273

20. Meidner, D. \& Vexler, B., A priori error estimates for space-time finite element discretization of parabolic optimal control problems. I: Problems without control constraints. SIAM J. Control Optim. 47 (2008), 1150-1177. Zbl1161.49026 MR2407012

21. Meidner, D. \& VeXler, B., A priori error estimates for space-time finite element discretization of parabolic optimal control problems. II: Problems with control constraints. SIAM J. Control Optim. 47 (2008), 1301-1329. Zbl1161.49035 MR2407017

22. SChUmACher, A., Die Wärmeleitungsgleichung auf bewegten Oberflächen. Master's thesis, Universität Freiburg, 2010.

23. Sinha, R. K. \& DEKA, B., Optimal error estimates for linear parabolic problems with discontinuous coefficients. SIAM J. Numer. Anal. 43 (2005), 733-749. Zbl1094. 65093 MR2177888

24. Tröltzsch, F., Optimal control of partial differential equations. Theory, procedures, and applications. (Optimale Steuerung partieller Differentialgleichungen. Theorie, Verfahren und Anwendungen.). Vieweg, 2005. Zb11142.49001

25. Ulbrich, M., Semismooth Newton methods for operator equations in function spaces. SIAM J. Optim. 13 (2003), 805-841. Zbl1033.49039 MR1972217

26. Vierling, M., Ein semiglattes Newtonverfahren für semidiskretisierte steuerungsbeschränkte Optimalsteuerungsprobleme. Master's thesis, Universität Hamburg, 2007. 